\title{
Doppler-Resolved Kinetics of Saturation Recovery
}

Damien Forthomme, ${ }^{a}$ Michael L. Hause, ${ }^{a \dagger}$ Hua-Gen Yu, ${ }^{a}$ Paul J. Dagdigian, ${ }^{b}$

Trevor J. Sears, ${ }^{a, c}$ and Gregory E. Hall ${ }^{a} *$

\begin{abstract}
${ }^{\mathrm{a} C h e m i s t r y ~ D e p a r t m e n t, ~ B r o o k h a v e n ~ N a t i o n a l ~ L a b o r a t o r y, ~ U p t o n, ~ N Y ~ 11973-5000 ~}$
${ }^{\mathrm{b}}$ Department of Chemistry, Johns Hopkins University, Baltimore, MD 21218

${ }^{c}$ Department of Chemistry, Stony Brook University, Stony Brook, NY 11794-3400
\end{abstract}

KEYWORDS speed-dependent relaxation, Doppler velocity selection, non-thermal kinetics, CN radical, rotational energy transfer, molecular dynamics

${ }^{\dagger}$ Present Address: Air Force Research Laboratory, Kirtland Air Force Base, NM 87117

*Corresponding author. Email: gehall@bnl.gov 


\section{ABSTRACT}

Frequency modulated laser transient absorption has been used to monitor the ground state rotational energy transfer rates of $\mathrm{CN}$ radicals in a double-resonance, depletion recovery experiment. When a pulsed laser is used to burn a hole in the equilibrium ground state population of one rotational state without velocity selection, the population recovery rate is found to depend strongly on the Doppler detuning of a narrow-band probe laser. Similar effects should be apparent for any relaxation rate process that competes effectively with velocity randomization. Alternative methods of extracting thermal rate constants in the presence of these non-thermal conditions are evaluated. Total recovery rate constants, analogous to total removal rate constants in an experiment preparing a single initial rotational level, are in good agreement with quantum scattering calculations, but are slower than previously reported experiments and show qualitatively different rotational state dependence between Ar and He collision partners. Quasi-classical trajectory studies confirm that the differing rotational state dependence is primarily a kinematic effect.

\section{Introduction}

The use of high resolution lasers to monitor the time evolution of rovibrational state distributions and their velocity distributions is common practice. Non-equilibrium velocity distributions can be monitored with sub-Doppler frequency resolution, for example, in photofragmentation, ${ }^{1,2,3}$ hot atom thermalization, ${ }^{4,5}$ or photoinitiated bimolecular collision products. ${ }^{6,7}$ The use of a Doppler shift for velocity selection to study velocity-dependent reaction cross sections has been applied to rotational energy transfer (RET) in excited states, ${ }^{8,9}$ and in 
Doppler-resolved double-resonance methods, ${ }^{10,11,12}$ where corrections for Doppler selection are commonly applied in careful kinetic studies of energy transfer. ${ }^{11,13,14}$ The competition among optical excitation, velocity-changing collisions, and inelastic collisions plays a central role in a substantial body of classic work on collisional effects in sub-Doppler saturation spectroscopy and speed-dependent collisional broadening. ${ }^{8,15,16,17,18,19}$ Observations similar to those in the present report have been noted in the context of inelastic and depolarizing collisions of the $A$ state of $\mathrm{CN}$ in the laboratory of Costen and McKendrick. ${ }^{20,21}$

For chemical kinetics measurements, velocity-specific detection can be an inconveniently specific tool, in the same way that rotationally resolved spectroscopic rate measurements can be complicated by changing non-thermal rovibrational distributions for reactions that compete with rotational thermalization. ${ }^{22}$ For relatively slow reactions performed at high enough pressures, fast rotational energy transfer can maintain thermal rotational distributions, and similar rates are observed for any rotational state. Similarly, if velocity randomization collisions compete effectively with those reactive or energy transfer processes that discriminate among velocities, the Doppler line shape will remain thermal, at the local temperature of the bath gas. Under these conditions, only the signal amplitude and not the kinetics will vary with Doppler detuning.

In contrast, our recent experiments to measure rotationally inelastic rates for ground state $\mathrm{CN}$ radicals by saturation recovery kinetics revealed a large, systematic variation in the signal recovery rates as a function of the Doppler detuning. The observations may seem at first surprising, since, unlike the earlier reports of initial Doppler selection and subsequent translational thermalization, ${ }^{8-14}$ the transient bleaching of our probed rotational states is performed without Doppler selection, yet the Doppler spectrum of the depletion, or "hole," shows rapid translational cooling in the initial stages of the return to equilibrium. This report 
characterizes the Doppler-resolved kinetics measured under these conditions, and the inelastic and elastic collisions that account for these effects.

Following a description of the experimental details of the double-resonance kinetic measurements in section 2, we describe the qualitative features of the saturation recovery experiment in section 3.1. An analysis based on a time-sequence of spectra is presented in section 3.2, and an alternate global analysis formulated in terms of speed-dependent rates is presented in section 3.3. Consistent thermal rate constants for total recovery due to rotational energy transfer through collisions with either $\mathrm{He}$ or Ar, and the photolytic precursor $\mathrm{CH}_{3} \mathrm{COCN}$ are obtained using either method. Section 4.1 includes a comparison of the present RET rates with previously published experimental and theoretical values. New quantum inelastic scattering calculations for $\mathrm{Ar}+\mathrm{CN}\left(X^{2} \Sigma^{+}\right)$were carried out as part of this work. The qualitatively different rotational-state dependence of RET total removal rate constants with $\mathrm{He}$ and Ar is discussed in section 4.2 with the help of some quasi-classical trajectory calculations. The connections between the present measurements and speed-dependent relaxation and velocity changing collisions are briefly discussed in section 4.3. An analysis of depolarization kinetics and saturation transfer kinetics, and a more thorough comparison with theory are reserved for a subsequent report.

\section{Experimental Methods}

Elastic and inelastic collisions of the $\mathrm{CN}$ radical $X^{2} \Sigma^{+}, v=0$ ground state were studied using a V-type optical-optical double resonance scheme to monitor the kinetics of ground state saturation recovery. Many features of the experiment have been described in our previous reports of subDoppler saturation spectroscopy ${ }^{23,24}$ and pressure broadening studies of the $\mathrm{CN} A-X$ transitions, ${ }^{25}$ using a frequency modulated, continuous wave Ti:sapphire laser to probe the $\mathrm{CN}$ radical. In this 
work, saturation was performed with a ns pulsed dye laser to deplete a chosen ground state level without Doppler selection. The experimental setup is shown in Figure 1. CN radicals were produced by $193 \mathrm{~nm}$ excimer laser photolysis of pyruvonitrile, $\mathrm{CH}_{3} \mathrm{COCN}$, (Aldrich, >90\% purity) in a $65 \mathrm{~cm}$ flow cell fitted with wedged, fused silica windows. The frequency-modulated (FM) probe laser was used to monitor $\mathrm{CN}$ radicals on selected rotational transitions near $900 \mathrm{~nm}$ in the $\mathrm{CN} A{ }^{2} \Pi \leftarrow X^{2} \Sigma^{+}(v=1 \leftarrow 0)$ band. The radio-frequency power into the modulator was adjusted to give a modulation index $\mathrm{M}=0.55$, corresponding to first-order sidebands each with about $8 \%$ of the residual carrier frequency power, as determined with an optical spectrum analyzer. The probe beam was collimated to a beam diameter of 1-2 mm, entirely contained within the much larger photolysis volume, produced by $\sim 5 \mathrm{~mJ}$ in a $1 \mathrm{~cm}^{2}$ pulse at $193 \mathrm{~nm}$. The bleach beam came from a YAG-pumped dye laser tuned between 620 and $625 \mathrm{~nm}$ to saturate selected rotational lines in the $\mathrm{CN} A^{2} \Pi \leftarrow X^{2} \Sigma^{+}(v=4 \leftarrow 0)$ band. The bleach beam pulse energy was typically adjusted to $1-1.5 \mathrm{~mJ} /$ pulse with polarization optics and then passed through a fused silica photoelastic modulator, synchronized with the YAG Q-switch timing to allow software control of its polarization during the scan.

The counterpropagating bleach and probe beams crossed at an angle of $0.5^{\circ}$, with the probed volume mostly contained within the larger bleached volume. Premixed samples of pyruvonitrile and $\mathrm{He}$ or Ar were prepared at dilutions between 1:10 and 1:100 and introduced to the sample cell through a mass flow controller. The inlet flow rate was maintained at $5 \mathrm{sccm}$ and the total steady-state pressure was adjusted with a throttled and liquid-nitrogen-trapped mechanical pump to give 10 mTorr partial pressure of the photolytic precursor at total pressures between 100 and 1000 mTorr, as monitored with a capacitance manometer. 
The optical receiver consisted of a fast Si photodiode (Hamamatsu S3883) viewing the probe beam transmitted through the sample cell. The amplified RF component of the photodiode signal was demodulated in an I\&Q demodulator, referenced to the local oscillator at $192 \mathrm{MHz}$. The in-phase (I) and quadrature (Q) components were low-pass filtered (Minicircuits SBLP-39) and averaged on a digital oscilloscope to give 4000-point transient waveforms spanning $20 \mu$ s. Typical scans used 100 excimer shot averaging for three cycles at each wavelength step of the probe laser: one with the bleach beam polarized parallel to the probe beam polarization, one with the bleach beam blocked with a shutter and a third with the bleach beam perpendicular to the probe beam polarization. A pair (I\&Q) of transient waveforms was archived for each of these three cases at 50-60 probe frequency steps of $\sim 80 \mathrm{MHz}$ across the Doppler profile, along with wavemeter data, for subsequent analysis.

\section{Results}

3.1. Photolysis, Saturation and Recovery. A typical raw saturation recovery signal at a probe frequency near the peak FM signal for a $\mathrm{CN}$ transition is shown in Figure 2 . The demodulated and low-pass filtered transient waveform is plotted against time, with the excimer photolysis laser firing $\sim 12 \mu$ s prior to the time origin, when the bleach laser fires. For this example, the probe laser is tuned to a maximum of the FM signal of the $\mathrm{P}_{2}(6)$ line of the (1-0) band, while the bleach laser is tuned to the center of the corresponding $\mathrm{P}_{2}(6)$ line of the (4-0) band. It can be seen that the initial signal at the time of photolysis is small and grows for several $\mu \mathrm{s}$, while both the rotational and translational distributions cool from hot nascent values, as investigated earlier. ${ }^{26}$ Fitting the time-delayed FM Doppler spectra shows effective translational temperatures decreasing from above $1300 \mathrm{~K}$ at early times down to about $300-320 \mathrm{~K}$ at the time 
of the bleach laser pulse. The initial rotational distribution of $\mathrm{CN}$ in $v=0$ can be characterized by a temperature near $1800 \mathrm{~K},{ }^{26}$ but at the pressures used in these experiments, it too becomes nearly stationary, near room temperature after 5-10 $\mu$ s. The thermal $\mathrm{CN}$ signal persists for over $100 \mu \mathrm{s}$, but is lost through slow reactions, diffusion and pump-out long before the next excimer pulse $100 \mathrm{~ms}$ later.

The inset to Figure 2 expands the time scale around the time of the bleach laser, and displays the fractional depletion signal at this probe frequency as a function of time for one polarization geometry. The depletion amplitude is obtained by subtracting the unbleached reference waveform from the bleached transient waveform. To correct for any variation in the excimer power or sample concentration between the reference and the bleached cycles at each step of the frequency scan, the reference waveform is scaled by the ratio of bleached to unbleached signals in a time gate immediately prior to the arrival of the bleach laser pulse, a scale factor typically between 0.98 and 1.02 . Each scan is initially processed to give a timedependent depletion amplitude for I and Q channels in each of the two polarization geometries, yielding FM depletion signal vs. time and frequency, for subsequent analysis described below. Division of the depletion amplitude by the slowly decaying unbleached reference signal yields the fractional depletion, which represents pure rotational redistribution of a normalized population, as illustrated in Figure 2.

The recovery kinetics are a measure of the redistribution of population among the rotational states of $\mathrm{CN}\left(X^{2} \Sigma^{+}, v=0\right)$ following a partial, permanent removal of the population of one rotational state due to the bleach laser. On the ns time scale of bleaching, the selected rotational level of the ground state is depleted to a fraction of its initial Boltzmann equilibrium population, while creating an excited state population in $v=4$ of the $A$ state equal to lost population of the 
ground state. The $A$ state radiative lifetime in $v=4$ is about $4 \mu \mathrm{s}^{27}$ with a Franck-Condon factor that limits the repopulation of $v=0$ in the $X$ state to only about $1 \%$ of the radiative relaxation from $v=4$ in the $A$ state,${ }^{28}$ and the vibrational relaxation in the $X$ state is also negligibly slow on the time scale of rotational energy transfer. Quenching by rare gas or the pyruvonitrile precursor will reversibly populate isoenergetic high vibrational levels of the $X$ state, ${ }^{27,29,30}$ which are metastable on the $\mu$ s time scale of the rotational energy transfer measurements. Consequently, the excited molecules are effectively removed irreversibly from the $v=0$ in the $X$ state, and the subsequent relaxation kinetics depends only on the rotational redistribution within this vibrational level and the much slower reactive and diffusive loss of the total ground state population.

The kinetics of depletion recovery, following the partial bleaching of a single rotational state from a thermalized distribution is isomorphic to the kinetics of relaxation of the same state, initially prepared in an otherwise empty rotational manifold. ${ }^{31}$ The kinetic master equation is the same and the initial conditions differ only by a sign change in the perturbation, and the addition of the stationary eigenvector (the Boltzmann rotational distribution). With appropriate normalization, the kinetics are identical and the same information can be extracted from either type of experiment.

The linearly polarized bleach laser creates an aligned excited state population, leaving behind a ground state hole with the complementary alignment. The probe signal thus depends on the relative polarization of bleach and probe lasers. The transient depletion recovery amplitudes for parallel $\left(I_{\|}\right)$and perpendicular $\left(I_{\perp}\right)$ polarizations can be written as

$$
\begin{aligned}
& I_{\|}(t, \omega)=C \quad g_{D}(\omega, t) A_{0}^{0}(t)\left(1+h^{(2)} A_{0}^{2}(t)\right) \\
& I_{\perp}(t, \omega)=C \quad g_{D}(\omega, t) A_{0}^{0}(t)\left(1-\frac{1}{2} h^{(2)} A_{0}^{2}(t)\right)
\end{aligned}
$$


where $\mathrm{C}$ is a constant including laser powers, optical path length, linestrength and instrumental factors; $h^{(2)}$ is the rank 2 depolarization factor ${ }^{32,33}$ for one-photon absorption, and $g_{D}(\omega, t)$ is a normalized Doppler lineshape function, generally time-dependent, but in this case negligibly dependent on the polarization. The alignment can be cancelled with the weighted sum $\left(I_{\|}+2 I_{\perp}\right)$ to yield a time-dependent signal proportional to the isotropic population depletion, $A_{0}^{0}(t)$. The polarization difference, $\left(I_{\|}-I_{\perp}\right)$, when normalized to the isotropic depletion, is proportional to the alignment moment of the hole, $A_{0}^{2}(\mathrm{t})$. We devote the present report to the analysis of the isotropic population change, $A_{0}^{0}(t)$, and defer treatment of the depolarization kinetics to a subsequent report.

3.2 Spectral evolution of the $\mathbf{C N}$ hole. The time-dependent spectrum of the isotropic depletion is initially thermal, with the same translational temperature as the $\mathrm{CN}$ population prior to saturation. Including power broadening, the effective line width of the multimode dye laser used for bleaching is large enough to saturate the transitions without detectable velocity selection. Successive time slices of the I and Q channel FM depletion amplitudes were fit to thermal lineshape functions, with an adjustable temperature, Doppler-integrated intensity (proportional to the total depletion), and a phase angle representing the complementary combinations of dispersion and absorption contributions to I and Q channel signals. The absorption-phase FM spectra resemble Gaussian first derivative lineshapes, and the dispersionphase FM spectra resemble second derivatives of the associated dispersion lineshapes, as described in further detail elsewhere. ${ }^{20,25,34,35}$ As an example of the time-dependent depletion spectra and the thermal fits, Figure 3 illustrates absorption- and dispersion- phase FM spectra in individual 5-ns time slices selected at 50, 150, and $250 \mathrm{~ns}$ following the bleach laser pulse. The 
data shown are for probing and bleaching $\mathrm{R}_{1}(0)$ rotational lines in a sample containing 590 mTorr of Ar and 10 mTorr of the precursor. I and Q channel FM depletion signals are polarization averaged and plotted as phase-rotated linear combinations using the optimized phase angle from the least squares fit. The best fit thermal absorption and dispersion lineshapes, shown as solid lines, share for each time slice a common temperature and a single adjustable amplitude, proportional to the Doppler-integrated depletion. Substantial translational cooling is observed on this timescale. The temperature of the hole drops $\sim 35 \mathrm{~K}$ per $100 \mathrm{~ns}$, which is the approximate 1/e time of the depletion recovery for this sample and rotational state.Some elementary remarks and further illustrations of the effects of narrow-band probing of relaxation kinetics when accompanied by translational cooling are presented in Supplement 1 of the Supplemental Information for this paper.

Figure 4 illustrates the time-dependent temperature and the corresponding total depletion recovery kinetics for a dense set of time slices from the same data set used in Figure 3 . The Doppler-integrated total depletion amplitudes are shown as linear (4a) and logarithmic (4b) plots for this example scan where the translational cooling is very clear. The spacing of displayed data points becomes sparser at longer times, as we have averaged over increasingly larger time bins in order to control the uncertainties in the fits to the signals of decreasing amplitude. Pressure broadening of the $A-X \mathrm{CN}$ transitions ${ }^{25}$ at a maximum pressure of 1 Torr will contribute a Lorentzian broadening with less than $1 \%$ of the Doppler width, and is not included in the present analysis.

Figure $4 \mathrm{a}$ shows a rapid initial translational cooling followed by slow reheating back to the ambient temperature. The depletion recovery kinetics shown in Figure $4 \mathrm{~b}$ and $4 \mathrm{c}$ are typical of many state-resolved energy transfer measurements ${ }^{13,36,37,38,39}$ where the initial decay is 
exponential, at a rate indicative of the total removal (recovery) process, but then decelerates as multiple collision effects start to become significant, and finally a new thermalized signal level is attained, once the population (depletion) has been rotationally equilibrated. Master equation treatments can relate a full matrix of state-to-state RET rate constants to the full multi-collision time evolution from an arbitrary initial condition, but the initial log slope of the decay of a directly populated state's population (or depletion) can be shown to give directly the total removal (recovery) rate without approximation. ${ }^{31}$

A set of depletion recovery measurements was made for seven rotational states at a series of concentrations of pyruvonitrile in $\mathrm{He}$ and in Ar, always adjusting the total pressure to maintain the partial pressure of pyruvonitrile at 10 mTorr. Transitions chosen were $R_{1}(0), P_{2}(4), R_{1}(4)$, $P_{2}(6), P_{2}(10), R_{1}(13)$ and $Q_{1}(17)$, using the corresponding transitions in the 4-0 and 1-0 bands for bleach and probe processes. The rotational states were chosen to span a range of thermally populated levels that could be probed and bleached without interference from nearby or blended lines. One pair of lines, $\mathrm{P}_{2}(4)$ and $\mathrm{R}_{1}(4)$, was chosen to probe independently the $\mathrm{F}_{1}$ and $\mathrm{F}_{2}(J=$ 5.5 and 4.5) spin-rotation levels of the $N=4$ rotational state, an example of a measurement that could not be done with $B-X$ spectroscopy, where the spin rotation doublets are unresolved at low $N$. No significant difference in the RET rates was observed between these two spin-rotation levels, as expected from the quantum scattering calculations.

A time series of thermal fits to the FM depletion signals, as depicted in Figures 3 and 4, was performed for each scan, and the time-dependent, Doppler-integrated, total depletion amplitudes were fit to a single exponential during the first $50 \%$ of the first time constant $(t<1 / 2 k)$. The initial decay rates were corrected for the slow decay of the reference signal observed in the absence of the saturation pulse. The slope of the corrected initial decay rates with respect to the 
He or Ar partial pressure produced the estimates of the total recovery rate constants listed in the third columns of Tables 1 and 2 , with $1 \sigma$ uncertainties derived from the scatter in the linear fits.

3.3 Speed-dependent relaxation rates. An alternate analysis method was developed that does not depend on independently adjusted temperature fits to each time slice, thereby avoiding the amplitude noise that is correlated with the poorly determined temperature at longer times as the intensity decreases. A global fit to each entire time and frequency-dependent FM depletion signal was implemented, based on an empirical functional form with only a few adjustable parameters, and an analytic means of extracting the initial decay rate, evaluated at the initial temperature.

The relaxation rate is expressed as an explicit function of the probe laser detuning, inspired by the increasing average relative collision velocity of thermal bath molecules with a Dopplerselected velocity group of probed molecules:

$$
k\left(x_{D}\right)=k_{0} \sqrt{1+b x_{D}{ }^{2}}
$$

Here, $x_{D}$ is the dimensionless Doppler shift

$$
x_{D}=\frac{\left(\omega-\omega_{0}\right) c}{\omega_{0} \sqrt{2 k_{B} T / m}}
$$

where $\omega-\omega_{0}$ is the detuning from line center in $\mathrm{cm}^{-1}$, and $m$ is the $\mathrm{CN}$ mass. The rate at linecenter is $k_{0}$, and $b$ is a dimensionless adjustable parameter to characterize the speed-dependence of the relaxation rate in a bath of constant temperature $T$. The functional form of Eq. (2) is motivated by the rms collision velocity of a Doppler selected particle colliding with a thermal target molecule. If the total inelastic cross-section is independent of velocity, and velocity changing collisions do not compete with inelastic collisions, and the collisions of $\mathrm{CN}$ with the 
undissociated precursor can be neglected, then the relaxation rate for the Doppler-selected velocity group would follow this form, with $b$ determined entirely by the masses of the collision partners. Allowing $b$ to float gives a single effective parameter to represent the speed dependence of the relaxation rate that subsumes all the ways these idealizations can be wrong. The adequacy of the representation will be assessed in section 4.3 below.

At each detuning $x_{D}$, the time-dependent relaxation signal is represented as

$$
A\left(x_{D}, t\right)=\left(e^{-k\left(x_{D}\right) t}+A_{\infty}\right) G_{0}\left(x_{D}\right) e^{-k_{\text {loss }} t}
$$

where $G_{0}\left(x_{D}\right)=\alpha e^{-x_{D}{ }^{2}}$ is a time-independent thermal Gaussian lineshape function, including a single global amplitude, $\alpha$, and characterizing the identical initial and final temperatures $\mathrm{T}$ through the implicit frequency scaling of $x_{D}$. The slow loss of total population during the rotational relaxation is included with the damping factor $e^{-k_{\text {loss }} t}$. The background loss rate, $k_{\text {loss }}$ varied between $2 \times 10^{4}$ and $7 \times 10^{4} \mathrm{~s}^{-1}$, as determined independently from the unbleached transient data recorded in each scan. The functional form of single exponential decay to a final offset is exact for a two-state reversible system returning to equilibrium, as if the bath of all unobserved states were an undifferentiated kinetic species with a single reverse rate to the probed level, and often provides a good, though not exact representation of total RET kinetics. ${ }^{40,41}$ By restricting the temporal region of interest to the initial stages of relaxation, prior to attainment of full equilibrium, the functional form is flexible enough to represent accurately the type of decelerating decay illustrated in Figure $4 \mathrm{c}$ for times as long as 2 or 3 time constants. The adjustable parameter $A_{\infty}$ can then be reinterpreted not as the true equilibrium Boltzmann population ratio of the detected state to the sum of all other states, but rather as an empirical deceleration parameter, representing the curvature of the initial log decay. 
One advantage of this functional form is that it represents a larger and more accurately determined data set, and one can analytically evaluate the thermally averaged decay rate at $\mathrm{t}=0$ from the global fit parameters $k_{0}, A_{\infty}$ and $b$.

$$
k_{\text {ave }}=\frac{k_{0} f(b)}{\left(1+A_{\infty}\right)}
$$

where the thermal average over the speed-dependent rate gives a correction factor

$$
f(b)=\frac{1}{\sqrt{\pi}} \int_{-\infty}^{\infty} e^{-x_{D}^{2}} \sqrt{1+b x_{D}^{2}} d x_{D}
$$

Over the range of interest in the present experiments, with $0<b<1$, this function is well described by the quadratic approximation $f(b) \approx 1.000+0.2385 b-0.0389 b^{2}$, which represents the factor by which the thermally averaged rate exceeds the rate for the cooler velocity group probed at line center.

A nonlinear least-squares fit was implemented in MATLAB, adjusting a single initial temperature, $T$, the parameters $k_{0}, A_{\infty}, b$, the line center of the transition, $\omega_{0}$, the overall signal amplitude, $\alpha$, and a single DC baseline offset constant, to model the frequency-dependent saturation recovery kinetics $A\left(x_{D}, t\right)$ according to Equation 4 . The parameters were optimized to minimize the squared difference between the experimental FM depletion signal in time and frequency and the model FM signal derived from $A\left(x_{D}, t\right)$ using the relation between absorption phase FM signals $A_{F M}\left(x_{D}\right)$ and the Doppler absorption spectrum $A\left(x_{D}\right)$ at each time:

$$
A_{F M}\left(x_{D}\right)=\left\{A\left(x_{D}+x_{R F}\right)-A\left(x_{D}-x_{R F}\right)\right\}\left(1-M^{2} / 8\right)+\left\{A\left(x_{D}+2 x_{R F}\right)-A\left(x_{D}-2 x_{R F}\right)\right\} M^{2} / 8
$$

Here $x_{R F}=\frac{\omega_{R F} c}{\omega_{0} \sqrt{2 k_{B} T / m}}$ is the modulation frequency $\omega_{\mathrm{RF}}$ expressed in the same normalized units as the Doppler shift, $x_{D}$. With the modulation frequency at $192 \mathrm{MHz}$ and a typical $\mathrm{CN}$ 
transition near $900 \mathrm{~nm}$ at $300 \mathrm{~K}, x_{R F}$ is near 0.4 . For the modulation index, $M=0.55$, used in the present measurements, the correction for second-order sidebands included in Eq. 7 is small, and the third-order correction (not included in Eq. 7) is negligible. ${ }^{35,42}$

An example of the resulting fits is illustrated in Figure 5, showing observed and calculated FM transient signals at different detunings, sampled from the saturation recovery measurements on the $\mathrm{P}_{2}(4)$ line in a sample containing Ar at total pressure of 200 mTorr. The entire FM signal at all frequencies and over the illustrated time window $\left(0<t<2 / k_{0}\right)$ was fit with the minimal set of parameters listed above. The data within $50 \mathrm{~ns}$ of the time origin is given no weight in the fit due to the finite bandwidth of the detection, and increased uncertainties associated with phase correction during and shortly after the rapid depletion. The larger deviations between observed and fit kinetics at early times in Figure 5 reflect this. The illustrated decay curves show average signals at detunings corresponding to the maximum of the FM signal and at larger detunings where the FM signal is about half as large. The solid lines represent cuts through the threedimensional global fit at the corresponding Doppler shifts, normalized to unit initial depletion amplitudes. It may be seen in this case that the decay is about $45 \%$ faster at the larger Doppler shift illustrated, which corresponds to a parameter $b$ of 0.56 for this rotational state and sample mixture (10 mTorr $\mathrm{CH}_{3} \mathrm{COCN}$ and 290 mTorr Ar).

The length of the time record included in the kinetic analysis using Eq. 4 introduces a modeldependent variation into the estimated initial rates that exceeds the statistical errors. Excluding data at times beyond two decay time constants allows $A_{\infty}$ to float above the actual asymptotic value and allows the initial slope and curvature of the logarithmic decay to be described with an accurate empirical function. Restricting the fits to times much less than one decay time constant is inappropriate, since then $k_{0}$ and $A_{\infty}$ are too closely correlated to be independently determined. 
At times of three or more time constants, the approach to final equilibrium is more nearly complete, and $A_{\infty}$ will be a good approximation of the final value, but the single exponential $k_{0}$ is then inadequate to represent the multi-exponential decay, and the model fits are systematically too slow at early times. Our preferred analysis includes data selected from the first two time constants of decay.

The thermally averaged, initial recovery rates defined by Eq. 5 are plotted against the partial pressure of $\mathrm{He}$ or Ar in Figure 6 for a sample of three rotational states. The slopes give the thermal rate constants for total recovery (removal) for the corresponding rotational state and collision partner, while the intercepts give the contribution due to the precursor, $\mathrm{CH}_{3} \mathrm{COCN}$ at a constant partial pressure of 10 mTorr. The lines included in Figure 6 are the result of fits that constrain the $\mathrm{He}$ and Ar lines for the same rotational state to share a common intercept. Note that the precursor makes a significant contribution to the RET, increasingly so at low rotational states. The rate vs. He pressure lines are nearly parallel for the three illustrated states, revealing differences almost entirely accounted for by the change in RET due to the precursor. In contrast, the corresponding lines for Ar show a significant change in slope among the three illustrated rotational states. The same analysis applied to the entire set of saturation recovery measurements leads to the thermally averaged initial recovery rates listed in the fourth column of Table 1 for Ar and Table 2 for He, and in the third column of Table 3 for $\mathrm{CH}_{3} \mathrm{COCN}$.

To confirm the large and strongly $N$-dependent $\mathrm{CN}$ RET rates for $\mathrm{CH}_{3} \mathrm{COCN}$, we performed several additional measurements with a range of $\mathrm{CH}_{3} \mathrm{COCN}$ partial pressures between 2 and 10 mTorr and a constant Ar partial pressure of 100 mTorr. The slopes of $\mathrm{k}_{\text {ave }}$ vs. the $\mathrm{CH}_{3} \mathrm{COCN}$ partial pressure for three rotational states investigated lead to the rates listed in column 4 of 
Table 3, which confirm the trends given by the intercepts of the more extensive $\mathrm{He}$ and Ardependent measurements.

The values of the speed-dependent parameters $b$ from the global fits are plotted for multiple rotational states as a function of $\mathrm{He}$ and Ar partial pressure in Figures $\mathrm{S} 4$ and S5 in the Supplemental Information. Error bars denote $1 \sigma$ uncertainties derived from the least-squares fit, although the variation with pressure and among rotational states is more irregular than these statistical errors, suggesting additional systematic errors in the determination of the speeddependent $\mathrm{b}$ parameters. Fortunately, the propagation of uncertainty in $b$ to the thermally averaged rate constants is modest, with a $50 \%$ uncertainty in $b$ resulting in only a $4 \%$ error in the corrected thermal rate constant for typical values of $b$ around 0.3 .

\section{Discussion}

4.1 Comparison of total RET rates with previous experiments and theory. The rate constants for total rotationally inelastic energy transfer measured in these studies differ from previously reported values by Fei et al. for both $\mathrm{He}^{37}$ and $\mathrm{Ar}^{43}$ collisions, as can be seen in Tables 1 and 2. Their experiments used stimulated emission pumping of selected rotational states of $\mathrm{CN}\left(X^{2} \Sigma^{+}, v=2\right)$, and measured the disappearance of the pumped level and the appearance of population in unpopulated levels as a function of delay time by pulsed laser induced fluorescence. Our rate constants are slower by as much as a factor of 2 compared to the previously published values, and in particular, show a qualitative difference in the rotational state dependence. The decrease in rate constant for increasing rotational levels observed for Ar collisions is replaced for He collisions by a near independence of the RET rate on rotational states (at least up to $\mathrm{N}=17$ ) in our measurements. What can cause these differences? Our measurements were performed with rotational states in $v=0$ compared to the previous 
measurements in $v=2$, but no strong variation in RET among nearby vibrational states is expected. $^{44,45}$ Our measurements resolve the $F_{1}$ and $F_{2}$ spin-rotation levels, so that our total recovery rates include an additional contribution from the nearly elastic spin-rotation statechanging collisions that are not part of the measurements with the unresolved spin-rotation doublets in the $B$-X LIF measurements - not a reason for our measured rate constants to be smaller than those measured by LIF. Because of the very good agreement of the state-to-state RET rate constants reported between the previous SEP/LIF experiments ${ }^{37}$ and the quantum scattering results of Lique et al. $^{45}$ and the reasonable expectation that the total removal rate constants should be decreasing at higher rotational levels due to exponential energy gap arguments, we searched hard for possible systematic problems with our method of FM spectroscopy in the depletion recovery mode for the determination of RET rate coefficients, and in particular, the quantitative consequences of the observed translational cooling on the extraction of rate coefficients. Following our analysis, we find that while the speed-dependent variations of the apparent recovery rates observed with narrow-band probing can vary by up to a factor of 2 depending on the detuning, our best estimates of the thermally averaged total RET rates remain significantly smaller than those of Fei et al. ${ }^{37,43}$ and display a qualitatively different trend with rotational state in the case of He only.

The previous comparisons of experiment and theory for $\mathrm{He}+\mathrm{CN}\left(X^{2} \Sigma^{+}\right)$collisions highlighted the even-odd alternation in state-to-state rate constants, noting a better agreement at higher rotational states, and speculating that the differences observed at lower rotational states could be attributed to deficiencies in the potential. ${ }^{45}$ To compare total rotationally inelastic rate constants from selected initial states, we have summed over final states of the calculated state-tostate thermal rate constants, as tabulated in the supplemental information of Lique et al. ${ }^{45}$ These 
calculated total removal rate constants indeed show much better agreement with the present depletion recovery measurements than with the SEP/LIF measurements. The final column of Table 1 shows these total RET rate coefficients from the Hibridon calculations of Lique et al. ${ }^{45}$ and Figure 7 summarizes the rotational state dependence of total inelastic rate constants for $\mathrm{CN}$ $\left(X^{2} \Sigma^{+}\right)$with $\mathrm{He}$ from multiple investigations, including our own quasi-classical trajectory studies, extended to higher rotational levels, briefly described in the following section. Figure 8 makes a similar comparison for the case of Ar collisions, including new quantum scattering calculations performed as part of this work. (For details on these calculations, see the Supplementary Information.)

We are led to suspect that the SEP/LIF rates ${ }^{37,43}$ may be overestimated due to an unrecognized contribution by the photolytic precursor, particularly so for the lower rotational states. The SEP/LIF rates were calculated from one concentration, typically $3.5 \%$, of $\mathrm{C}_{2} \mathrm{~N}_{2}$ and a single pressure, neglecting possible contributions of the precursor to the total decay rate. While $\mathrm{C}_{2} \mathrm{~N}_{2}$ is unlikely to be as efficient as our precursor, $\mathrm{CH}_{3} \mathrm{COCN}$, at enhancing the total RET rates, we note that we would substantially overestimate of the recovery rate constant, particularly for low rotational states, if we simply divided the observed rate by the partial pressure of $\mathrm{He}$ or Ar for $3.5 \%$ mixtures, rather than evaluating the slope with respect to the $\mathrm{He}$ or $\mathrm{Ar}$ partial pressure. We are no longer able to procure $\mathrm{C}_{2} \mathrm{~N}_{2}$ for tests in our laboratory, although previous RET measurements ${ }^{46}$ of $\mathrm{CN}\left(B^{2} \Sigma^{+}\right)$with $\mathrm{He}$ and $\mathrm{C}_{2} \mathrm{~N}_{2}$ collision partners show total RET rate constants nearly independent of rotational states for $\mathrm{He}$, while the $\mathrm{C}_{2} \mathrm{~N}_{2}$ rate constants were larger and strongly increasing at lower rotational states, a pattern qualitatively similar to what we observe for $\mathrm{He}$ and $\mathrm{CH}_{3} \mathrm{COCN}$ collisions with $\mathrm{CN}\left(X^{2} \Sigma^{+}\right)$. Earlier studies in the Wittig laboratory of rotational relaxation from very high rotational states of $\mathrm{CN}\left(X^{2} \Sigma^{+}\right)$pointed to a 
qualitative difference between $\mathrm{He}$ and $\mathrm{Ar}$ as a collision partner. ${ }^{47}$ Following $193 \mathrm{~nm}$ photodissociation of $\mathrm{BrCN}$, rotational levels of $\mathrm{CN}$ above 50 were found to be metastable in Ar, yet efficiently thermalized by He, indicating that the RET rates fall off at high rotational states much more slowly for He than for Ar collisions. ${ }^{47}$

Modelers who use experimental or theoretical energy transfer rate constants in astrophysics, ${ }^{48}$ combustion diagnostics, and non-thermal plasma diagnostics ${ }^{49}$ should be alert to these new rate constants, which appear fully consistent with the quantum scattering calculations.

\subsection{Kinematic differences between $\mathrm{Ar}$ and He collision partners. Following many} investigations into rotational energy transfer, a general trend for high rotational states to become increasingly resistant to state-changing collisions is expected, as the minimum energy gap between rotational states increases. ${ }^{50,51}$ Adiabaticity arguments can be invoked: as the time scale for rotation decreases relative to the timescale of the collisional encounter, a collisionally sudden limit at low rotation evolves toward a rotationally averaged and thus more isotropic interaction at high rotational states, suppressing the inelastic probabilities. ${ }^{9,51,52}$ In qualitative terms, the slower Ar collisions should reach this region of decreasing rotationally inelastic cross sections at lower rotational states than for He collisions, yet the rate constants must eventually decrease for thermal He collisions at high enough rotational states. Quantitatively, the details of the interaction potential will also matter. The excellent agreement between our experimental rates and the quantum scattering calculations on an ab initio surface with frozen $\mathrm{CN}$ bond length should not be surprising, but the scattering calculations are silent on the question of dynamics vs. kinematics, i.e., the relative importance of the interaction potential and the mass combinations. Is the modest difference in the interaction potential for $\mathrm{Ar}$ and He interaction with $\mathrm{CN}\left(X^{2} \Sigma^{+}\right)$at all important, or can the effects be primarily attributed simply to the differences in collision 
timescales? The dramatic differences between He and Ar observed by Hay et al. ${ }^{47}$ in the relaxation of very high rotational states of $\mathrm{CN}\left(X^{2} \Sigma^{+}\right)$encouraged us to explore the behavior at higher rotational levels.

Most features of RET are well described by quasi-classical trajectory (QCT) methods, ${ }^{53,54,55}$ which do not suffer from rapidly increasing computational effort at high energies and high angular momentum states. We therefore implemented a classical rigid rotor trajectory $\operatorname{program}^{56}$ to explore independent effects of the reduced mass and the potential in this RET process. A brief description of the QCT calculations is included in the Supplemental Information. Comparisons were made between total rotationally inelastic rate constants as a function of initial rotational level for $\mathrm{CN}\left(X^{2} \Sigma^{+}\right)+$He and hypothetical systems with the same potential $^{45}$ but replacing the mass of He with the mass of Ne or Ar. Another set of calculations used a CN $\left(X^{2} \Sigma^{+}\right)+$Ar potential $^{20}$ with the collider mass again chosen from the masses of He, $\mathrm{Ne}$ and Ar. The calculations with realistic masses for both the $\mathrm{CN}+\mathrm{He}$ and $\mathrm{CN}+\mathrm{Ar}$ cases agree very well with the quantum scattering calculations on the same potentials for initial rotational states $\mathrm{N}<18$, if the threshold angular momentum change for classifying collisions as inelastic is set to $\pm \hbar$.

The continuation to higher initial rotational levels that were not considered in the quantum scattering calculations can be seen as calculated QCT points in Figures 7 and 8. A continuously decreasing inelastic rate constant with increasing rotation is seen for Ar, as expected, while He shows a nearly flat region between $\mathrm{N}=3$ and 20 before beginning a more gradual decrease in rate constant at higher initial rotational states. Artificially changing the interaction potential from $\mathrm{CN}$ $\left(X^{2} \Sigma^{+}\right)+$He to the $\mathrm{CN}\left(X^{2} \Sigma^{+}\right)+$Ar potential, while keeping the collider mass fixed at 4 nearly doubles the rate constants but without significantly changing the rotational state dependence of 
the rate. The doubling of the rate constant is primarily due to the larger repulsive radius of Ar compared to $\mathrm{He}$, which increases the cross sections accordingly. Comparing the same two potentials with the mass fixed at 40 shows a different rotational state dependence, falling off more steeply at high rotational levels, but again with a state dependence independent of the mass, but with the overall rates approximately doubled in the $\mathrm{CN}\left(X^{2} \Sigma^{+}\right)+\operatorname{Ar}$ potential. When comparing the real $\mathrm{CN}\left(X^{2} \Sigma^{+}\right)+$Ar system with the real $\mathrm{CN}\left(X^{2} \Sigma^{+}\right)+$He system, the increase in the cross sections is compensated by the reduction in the mean relative velocities to make the rate constants similar. In this case, where the interaction potentials have similar repulsive gradients and only weakly attractive van der Waals minima, differing mostly in the range of the repulsive wall, the QCT studies support the view that kinematic effects are dominant in such atom-diatom inelastic encounters. ${ }^{57}$

\subsection{Speed Dependent Effects in energy transfer}

The relaxation rate at a given Doppler shift can be described as a non-thermal average over the speed-dependent inelastic cross section, with the relative velocity distribution biased by one Doppler-selected velocity component of the probed species. For a Doppler-selected velocity group of the absorbing molecule $\mathrm{CN}$ with a specific Doppler shift corresponding to $\mathrm{z}$-component of velocity for the absorber $v_{\mathrm{z}}$ the conditional probability of relative collision velocity $v_{r}$ is given by $^{19}$

$$
f\left(v_{r} \mid v_{z}\right)=2 \frac{v_{r} \sqrt{\alpha}}{v_{0}^{2} \sqrt{\pi}} \exp \left(-\frac{v_{r}{ }^{2}}{v_{0}^{2}}\right) \times \int_{-v_{r}}^{v_{r}} \exp \left[\frac{u^{2}-\alpha\left(v_{z}-u\right)^{2}}{v_{0}^{2}}\right] d u
$$

where $v_{0}=\left[2 k T\left(m_{a}+m_{p}\right) / m_{a} m_{p}\right]^{1 / 2} \equiv \sqrt{2 k T / \mu}$ is the most probable relative velocity at temperature $T ; m_{a}$ and $m_{p}$ are the masses of the absorber $(\mathrm{CN})$ and the perturber (He or Ar), and 
$\alpha=1+m_{p} / m_{a}$ (correcting a typographical error in reference [19], in which $m_{p}$ and $m_{a}$ were reversed). An equivalent expression for the conditional collision velocity was given by Smith, et al. ${ }^{9}$ which has been used for the analysis of Doppler-selected excitation studies:

$$
f\left(v_{r} \mid v_{z}\right)=\frac{v_{r}}{2 \sqrt{1+r}} \exp \left(\frac{-\left[v_{r}^{2}-(1+r) v_{z}^{2}\right]}{2(1+r)}\right) \times\left[\operatorname{erf}\left(\frac{v_{r}+(1+r) v_{z}}{\sqrt{2 r(1+r)}}\right)+\operatorname{erf}\left(\frac{v_{r}-(1+r) v_{z}}{\sqrt{2 r(1+r)}}\right)\right],
$$

with $r=m_{a} / m_{p}$ and both $v_{r}$ and $v_{z}$ normalized to a reference speed of $\sqrt{k T / m_{a}}$, (correcting a typographical error in the argument to the exponential function in Eq. 3a of ref 9).

The Doppler-selected rate at temperature $\mathrm{T}$ will then vary with the Doppler shift according to

$$
k\left(v_{z}\right)=\int_{0}^{\infty} v_{r} \sigma\left(v_{r}\right) f\left(v_{r} \mid v_{z}\right) d v_{r}
$$

where $\sigma\left(v_{r}\right)$ is the rotationally inelastic cross section for a relative velocity $v_{r}$. To compare with the measured Doppler-resolved relaxation rates, we can use Eq. 10 to perform non-thermal averages of the speed-dependent cross sections from the quantum scattering calculations, as outlined in the Supplementary Information. Figure 9a illustrates the calculated speed-dependent total rotationally inelastic cross sections for three of the rotational states measured in this work. Performing the non-thermal averages using Eq. 10 gives theoretical curves for the corresponding Doppler-resolved relaxation rates, for selected rotational states of $\mathrm{CN}\left(X^{2} \Sigma^{+}, v=0\right)$ colliding with Ar, illustrated as solid lines in Figure 9b.

Despite the substantially different forms of the speed-dependent cross sections, after averaging, the Doppler-shift resolved rates have a less distinctive variation, all of which can be quite well described by the empirical form of Eq. 2, used in the fitting of experimental data. The dashed lines superimposed on the numerical non-thermal averaged rates in Figure $9 \mathrm{~b}$ are the best-fit representations of the calculated Doppler-shift resolved rates adjusting $k_{0}$ and $b$ to 
represent the rates for probed Doppler shifts up to $1500 \mathrm{~m} / \mathrm{s}$. Table 4 summarizes the theoretical values of $k_{0}$ and $b$ determined in this way from the non-thermal averages of total rotationally inelastic rates of selected rotational states of $\mathrm{CN}\left(X^{2} \Sigma^{+}\right)$in collision with $\mathrm{Ar}$ and with He. As expected, the speed-dependence, parameterized by $b$ is smaller for He than for Ar. The rotational state dependence of $b$ reflects a variation in the shape of the speed-dependent cross sections. The values of $k_{0}$ are less than the unbiased thermal rate constants, by $10-15 \%$ in Ar, and a smaller margin in He.

Some qualitative features of these results can be compared to experiment, although the experimental measurement of the Doppler-resolved saturation recovery rates, as illustrated in Supplemental Figures S4 and S5 are noisy and affected by additional factors not included in the quantum scattering calculations. The error bars shown in the figures are $1 \sigma$ statistical errors from the global fits, but the scatter with respect to variations in pressure and rotational state indicates additional systematic errors associated with extracting second-order effects from imperfect data. There is a general trend toward larger $b$ values at lower rare gas pressures, as the precursor concentration is increasing and contributes disproportionately to the observed speeddependence. At the lower concentrations of precursor (higher total pressure), the trend toward smaller $b$ values for He collisions compared to Ar is confirmed, as the selected $\mathrm{CN}$ velocity makes less of a contribution to the total relative velocity for collisions with light targets. Only the extrapolation to high dilution gives the intrinsic rare gas speed-dependence, and at the high pressure, high dilution end of our measurement range the absolute rates are faster, closer to our limiting time resolution, making our determination of the speed dependence more uncertain there. 
A final and subtle complication in the interpretation of the Doppler-shift dependent saturation recovery rates is the influence of velocity-changing collisions. The molecules probed at a specific Doppler detuning will have an instantaneous velocity component along the probe beam direction selected by the laser frequency. In the presence of velocity randomizing collisions, however, the probed velocity groups are not persistent and uncoupled, and the relaxation rate will include not only the velocity-averaged inelastic rate of the probed velocity group, but also any net growth or decay due to velocity changing collisions, even during the time corresponding to a small fraction of the first inelastic collision. The competition between elastic and inelastic collisions with both rare gas and a precursor can add significant complexity to the concentration dependence and initial state dependence of the Doppler-resolved relaxation rates. The more effectively velocity randomization competes with inelastic collisions, the less deviation from thermal Gaussian line shapes will occur during the population thermalization, and the recovery rate will be more independent of the Doppler shift, leading to reduced values of $b$. The calculated non-thermal average recovery rates using Eq. 10 should thus be considered upper bounds to the speed-dependence, obtained by neglecting the effects of velocity-changing collisions. When experimental values of $b$ exceed the theoretical values, we suspect a continued contribution from inelastic collisions with the heavy precursor. Future experiments measuring narrow-band saturation recovery kinetics following sub-Doppler hole burning should distinguish between inelastic and elastic velocity-changing collisions that contribute to the spectral evolution of the depletion signal. 


\section{Conclusions}

This work demonstrates the use of transient FM spectroscopy to measure ground state depletion recovery kinetics in thermal gas phase samples containing $\mathrm{CN}$ radicals. Polarized absorption signals can be measured with sufficient dynamic range and fidelity to determine thermal rate constants for rotational energy transfer at pressures low enough to access the singlecollision time scale. Total rotationally inelastic rate constants show a dependence on the initial rotational state that is surprisingly different for collisions of $\mathrm{CN}(\mathrm{X})$ with $\mathrm{He}$ vs. Ar, in contrast to some previously reported experiments, but in good agreement with quantum scattering calculations.

Fast processes that compete effectively with velocity thermalization will be characterized by variable kinetics that depend on the Doppler detuning of a narrow band probe laser. Even when the initial pulsed perturbation to an initially thermalized system is performed with unbiased velocity selection, the Doppler-resolved probe process reveals speed-dependent reaction rates and a spontaneous cooling of the translational distribution, until velocity-changing collisions reestablish the Maxwell-Boltzmann velocity distribution at the ambient temperature. The visibility of the speed-dependent kinetic signature will depend on the mass ratio of the probed molecule and the collision partner, the variation of the cross section with the collision velocity, and the competition with elastic velocity-randomizing collisions. 


\section{Table 1.}

Total rotationally inelastic rate constants* for $\mathrm{CN}\left(X^{2} \Sigma^{+}\right)+\mathrm{He}$

\begin{tabular}{|c|c|c|c|c|c|}
\hline $\begin{array}{c}\text { Initial } \\
\mathrm{N}\end{array}$ & $\mathrm{F}_{1} / \mathrm{F}_{2}$ & $\mathrm{k}_{\mathrm{RET}}{ }^{\mathrm{a}}$ & $\mathrm{k}_{\mathrm{RET}}{ }^{\mathrm{b}}$ & $\begin{array}{c}\text { SEP/LIF }(v=2)^{\mathrm{c}} \\
\left(\text { unresolved } \mathrm{F}_{1}+\mathrm{F}_{2}\right)\end{array}$ & Calculated $^{\mathrm{d}}$ \\
\hline 0 & $\mathrm{~F}_{1}$ & $2.8(2)$ & $3.0(2)$ & & 3.17 \\
\hline 4 & $\mathrm{~F}_{2}$ & $1.9(2)$ & $2.1(3)$ & $4.00(20)$ & 2.73 \\
\hline 4 & $\mathrm{~F}_{1}$ & $2.0(2)$ & $2.1(3)$ & & 2.71 \\
\hline 6 & $\mathrm{~F}_{2}$ & $2.5(1)$ & $2.6(1)$ & & 2.7 \\
\hline 10 & $\mathrm{~F}_{2}$ & $2.3(1)$ & $2.4(2)$ & $3.61(10)$ & 2.63 \\
\hline 11 & & & & & 2.6 \\
\hline 13 & $\mathrm{~F}_{1}$ & $2.3(1)$ & $2.5(2)$ & $3.06(18)$ & 2.37 \\
\hline 17 & $\mathrm{~F}_{1}$ & $2.4(1)$ & $2.5(1)$ & $2.07(16)$ & \\
\hline 24 & & & & & \\
\hline 31 & & & & & \\
\hline
\end{tabular}

* Rate constants in units of $10^{-10} \mathrm{~cm}^{3} \mathrm{molec}^{-1} \mathrm{~s}^{-1}$.

a. Rate constants based on pressure dependence of single exponential fits to early time thermal population fits. The numbers in parentheses represent $1 \sigma$ statistical uncertainties in the preceding digits.

b. Rate constants based on pressure dependence of $298 \mathrm{~K}$ average of velocity-dependent initial rates from global fits to spectral data during the first two time constants of decay.

c. From ref [37], assuming $300 \mathrm{~K}$ for conversion from (Torr $\cdot \mathrm{ns})^{-1}$

d. Quantum scattering calculations ${ }^{45}$ using Hibridon code. ${ }^{58}$ 
Table 2.

Total rotationally inelastic rate constants* for $\mathrm{CN}\left(X^{2} \Sigma^{+}\right)+\mathrm{Ar}$

\begin{tabular}{|c|c|c|c|c|c|}
\hline $\begin{array}{c}\text { Initial } \\
\mathrm{N}\end{array}$ & $\mathrm{F}_{1} / \mathrm{F}_{2}$ & $\mathrm{k}_{\mathrm{RET}}{ }^{\mathrm{a}}$ & $\mathrm{k}_{\mathrm{RET}}^{\mathrm{b}}$ & $\begin{array}{c}\text { SEP/LIF }(v=2)^{\mathrm{c}} \\
\left(\text { unresolved } \mathrm{F}_{1}+\mathrm{F}_{2}\right)\end{array}$ & Calculated $^{\mathrm{d}}$ \\
\hline 0 & $\mathrm{~F}_{1}$ & $3.9(2)$ & $4.2(2)$ & & 4.73 \\
\hline 4 & $\mathrm{~F}_{2}$ & $2.5(2)$ & $2.8(3)$ & \multirow{2}{*}{$5.24(15)$} & 3.51 \\
\hline 4 & $\mathrm{~F}_{1}$ & $2.6(2)$ & $2.9(3)$ & & 3.47 \\
\hline 6 & $\mathrm{~F}_{2}$ & $2.5(2)$ & $2.8(2)$ & & 3.12 \\
\hline 10 & $\mathrm{~F}_{2}$ & $2.3(2)$ & $2.4(2)$ & & 2.72 \\
\hline 11 & & & & $4.06(31)$ & 2.60 \\
\hline 13 & $\mathrm{~F}_{1}$ & $2.1(1)$ & $2.2(2)$ & & 2.35 \\
\hline 17 & $\mathrm{~F}_{1}$ & $1.6(1)$ & $1.6(1)$ & & 1.81 \\
\hline 24 & & & & $2.42(46)$ & \\
\hline 31 & & & & $2.11(25)$ & \\
\hline
\end{tabular}

* Total removal (recovery) rate constants in units of $10^{-10} \mathrm{~cm}^{3}$ molec $^{-1} \mathrm{~s}^{-1}$.

a. Rate constants based on pressure dependence of single exponential fits to early time thermal population fits. The numbers in parentheses represent $1 \sigma$ statistical uncertainties in the preceding digits.

b. Rate constants based on pressure dependence of $298 \mathrm{~K}$ average of velocity-dependent initial rates from global fits to spectral data during the first two time constants of decay.

c. From ref [43], assuming $300 \mathrm{~K}$ for conversion from (Torr $\cdot \mathrm{ns})^{-1}$

d. This work, quantum scattering calculations using Hibridon code ${ }^{58}$ 


\section{Table 3.}

Total rotationally inelastic rate constants* for $\mathrm{CN}\left(X^{2} \Sigma^{+}\right)+\mathrm{CH}_{3} \mathrm{COCN}$

\begin{tabular}{|c|c|c|c|}
\hline$N$ & $\mathrm{~F}_{1} / \mathrm{F}_{2}$ & $\mathrm{k}_{\mathrm{RET}}^{\mathrm{a}}$ & $\mathrm{k}_{\mathrm{RET}}^{\mathrm{b}}$ \\
\hline 0 & $\mathrm{~F}_{1}$ & $95(5)$ & $78(3)$ \\
\hline 4 & $\mathrm{~F}_{2}$ & $75(8)$ & -- \\
\hline 4 & $\mathrm{~F}_{1}$ & $80(7)$ & -- \\
\hline 6 & $\mathrm{~F}_{2}$ & $43(3)$ & $34(1)$ \\
\hline 10 & $\mathrm{~F}_{2}$ & $32(4)$ & -- \\
\hline 13 & $\mathrm{~F}_{1}$ & $25(4)$ & $8.2(6)$ \\
\hline 17 & $\mathrm{~F}_{1}$ & $19(3)$ & \\
\hline
\end{tabular}

* Rate constants in units of $10^{-10} \mathrm{~cm}^{3}$ molec $^{-1} \mathrm{~s}^{-1}$. The numbers in parentheses represent $1 \sigma$ statistical uncertainties in the preceding digits.

${ }^{\mathrm{a}}$ Derived from common intercepts of $\mathrm{He}$ and Ar dependent rates at 10 mTorr $\mathrm{CH}_{3} \mathrm{COCN}$

${ }^{b}$ Derived from $\mathrm{CH}_{3} \mathrm{COCN}$ pressure dependence of initial recovery rates at fixed 100 mTorr $\mathrm{Ar}$ 


\section{Table 4.}

Theoretical Doppler-shift dependence of total rotationally inelastic rate constants for selected rotational states of $\mathrm{CN}\left(X^{2} \Sigma^{+}\right)+\mathrm{Ar}$ and He, at $298 \mathrm{~K}$. Fit to $k\left(x_{D}\right)=k_{0} \sqrt{1+b x_{D}{ }^{2}}$.

\begin{tabular}{|c|c|c|c|c|}
\hline $\mathrm{N}\left(\mathrm{F}_{1}\right)$ & $k_{0}\left(10^{-10} \mathrm{~cm}^{3} \mathrm{~mol}^{-1} \mathrm{~s}^{-1}\right)$ & $b$ & $k_{0}\left(10^{-10} \mathrm{~cm}^{3} \mathrm{~mol}^{-1} \mathrm{~s}^{-1}\right)$ & $b$ \\
\hline 0 & 3.08 & 0.083 & 4.66 & 0.145 \\
\hline 2 & 2.68 & 0.092 & 3.92 & 0.190 \\
\hline 4 & 2.63 & 0.090 & 3.31 & 0.272 \\
\hline 6 & 2.61 & 0.089 & 2.89 & 0.334 \\
\hline 8 & 2.59 & 0.089 & 2.68 & 0.349 \\
\hline 10 & 2.54 & 0.091 & 2.49 & 0.379 \\
\hline 12 & 2.49 & 0.095 & 2.25 & 0.447 \\
\hline 14 & 2.41 & 0.100 & 1.97 & 0.573 \\
\hline 16 & 2.33 & 0.105 & 1.66 & 0.780 \\
\hline
\end{tabular}




\section{Supporting Information}

S1. Temperature Dependence of FM Line Shapes

S2. Quantum Scattering Calculations of Rotationally Inelastic Collisions of $\mathrm{CN}(X)$ by Rare Gases

S3. Quasi-Classical Trajectory Calculations of Rotationally Inelastic Collision of $\mathrm{CN}(X)$ by Rare Gases

S4. Supplemental Figures S4 and S5, illustrating pressure-dependent values of speed-dependent relaxation parameters $b$ for $\mathrm{He}$ and $\mathrm{Ar}$ and an assortment of $\mathrm{CN}$ rotational states.

This material is available free of charge via the Internet at http://pubs.acs.org

\section{Author's Contributions}

DF performed most of the experimental work, analyzed the data, and performed the QCT calculations. Preliminary versions of the experimental work and analysis were done by MLH. HGY provided code and consultations on the QCT calculations. PJD performed the quantum scattering calculations, wrote the corresponding sections of the paper, and participated in discussions of the work. TJS participated in planning and discussions of the work. GEH designed the experiments, participated in the analysis and wrote most of the paper. All authors have given approval to the final version of the manuscript.

\section{Acknowledgements}

This work was carried out at Brookhaven National Laboratory under Contracts No. DE-AC0298CH10886 and DE-SC0012704 with the U.S. Department of Energy, Office of Science, and supported by its Division of Chemical Sciences, Geosciences, and Biosciences within the Office of Basic Energy Sciences. The theoretical portion of this work performed at JHU was supported 
by the U.S. Department of Energy, Office of Science, Basic Energy Sciences, under Award No. DESC0002323. We are grateful to George McBane and Millard Alexander for helpful discussions. 


\section{References}

1. Schmiedl, R.; Dugan, H.; Meier, W.; Welge, K. H., Laser Doppler spectroscopy of atomic hydrogen in the photodissociation of HI. Z. Phys. A: Atoms and Nuclei 1982, 304, 137142.

2. Gericke, K. H.; Klee, S.; Comes, F. J.; Dixon, R. N., Dynamics of $\mathrm{H}_{2} \mathrm{O}_{2}$ photodissociation: $\mathrm{OH}$ product state and momentum distribution characterized by sub-Doppler and polarization spectroscopy. J. Chem. Phys. 1986, 85, 4463-4479.

3. Nadler, I.; Mahgerefteh, D.; Reisler, H.; Wittig, C., The $266 \mathrm{~nm}$ photolysis of ICN: Recoil velocity anisotropies and nascent $\mathrm{E}, \mathrm{V}, \mathrm{R}, \mathrm{T}$ excitations in the $\mathrm{CN}+\mathrm{I}\left({ }^{2} \mathrm{P}_{3 / 2}\right)$ and $\mathrm{CN}+\mathrm{I}\left({ }^{2} \mathrm{P}_{1 / 2}\right)$ channels. J. Chem. Phys. 1985, 82, 3885.

4. Park, J.; Shafer, N.; Bersohn, R., The time evolution of the velocity distribution of hydrogen atoms in a bath gas. J. Chem. Phys. 1989, 91, 7861-7871.

5. Cline, J. I.; Taatjes, C. A.; Leone, S. R., Diode laser probing of $\mathrm{I}^{*}\left({ }^{2} \mathrm{P}_{1 / 2}\right)$ Doppler profiles: time evolution of a fast, anisotropic velocity distribution in a thermal bath. J. Chem. Phys. 1990, $93,6543-6553$.

6. Hershberger, J. F.; Hewitt, S. A.; Sarkar, S. K.; Flynn, G. W.; Weston, R. E., Quantum state-resolved study of pure rotational excitation of $\mathrm{CO}_{2}$ by hot atoms. J. Chem. Phys. 1989, 91, 4636-4642.

7. Brouard, M.; Duxon, S. P.; Enriquez, P. A.; Sayos, R.; Simons, J. P., Velocity-aligned photofragment dynamics: Stereodynamics in the reaction $\mathrm{O}\left({ }^{1} \mathrm{D}\right)+\mathrm{N}_{2} \mathrm{O} \rightarrow \mathrm{NO}+\mathrm{NO}$ J. Phys. Chem. 1991, 95, 8169-8174.

8. Phillips, W. D.; Pritchard, D., Velocity selection by Doppler shift: a general method for studying excited-state collisions. Phys. Rev. Lett. 1974, 33, 1254-1257. 
9. Smith, N.; Scott, T. P.; Pritchard, D. E., Velocity dependence of rotationally inelastic collisions: ${ }^{7} \mathrm{Li}_{2} *\left(\mathrm{~A}{ }^{1} \Sigma\right)+\mathrm{Ne}, \mathrm{Ar}$, and Xe. J. Chem. Phys. 1984, 81, 1229-1247.

10. Collins, T. L. D.; McCaffery, A. J.; Richardson, J. P.; Wynn, M. J., Spectroscopic determination of the state-to-state differential cross-section by velocity selected doubleresonance. Phys. Rev. Lett. 1993, 70 (22), 3392-3395.

11. Smith, T. C.; Hostutler, D. A.; Hager, G. D.; Heaven, M. C.; McBane, G. C., State-tostate rotational rate constants for $\mathrm{CO}+\mathrm{He}$ : Infrared double resonance measurements and simulation of the data using SAPT theoretical potential energy surface. J. Chem. Phys. 2004, $120,2280-2295$.

12. Abel, B.; Coy, S. L.; Klaassen, J. A.; Steinfeld, J. I., State-to-state rotational energy transfer measurements in the $v_{2}=1$ state of ammonia by infrared-infrared double resonance. $J$. Chem. Phys. 1992, 96, 8236-8250.

13. Phipps, S. P.; Smith, T. C.; Hager, G. D.; Heaven, M. C.; McIver, J. K.; Rudolph, W. G., Investigation of the state-to-state rotational relaxation rate constants for carbon monoxide using infrared double resonance. J. Chem. Phys. 2002, 116, 9281-9292.

14. Stewart, B. A.; Stephens, T. N.; Lawrence, B. A.; McBane, G. C., Rotational energy transfer in $\mathrm{Ne}_{-} \mathrm{Li}_{2}(\mathrm{~A}, \mathrm{v}=0)$ : Comparison of experimental data and results from classical and quantum calculations. J. Phys. Chem. A 2010, 114, 9875-9885.

15. Mattick, A. T.; Sanchez, A.; Kurnit, N. A.; Javan, A., Velocity dependence of collisionbroadening cross section observed in an infrared transition of $\mathrm{NH}_{3}$ gas at room temperature. Applied Physics Letters 1973, 23, 675-678.

16. Mattick, A. T.; Kurnit, N. A.; Javan, A., Velocity dependence of collision broadening cross sections in $\mathrm{NH}_{3}$. Chem. Phys. Lett. 1975, 38, 176-180. 
17. Letokhov, V. S., Saturation Spectroscopy. In High-Resolution Laser Spectroscopy, Shimoda, K., Ed. Springer-Verlag: Berlin, 1976; pp 95-171.

18. Coy, S. L., Speed dependence of microwave rotational relaxation rates. J. Chem. Phys. 1980, 73, 5531-5555.

19. Buffa, G.; Carocci, S.; DiLieto, A.; Minguzzi, P.; Quochi, F.; Tarrini, O.; Tonelli, M., Speed dependence of pressure broadening in molecular rotational spectra using a novel technique. Phys. Rev. Lett. 1995, 74, 3356-3359.

20. McGurk, S. J.; McKendrick, K. G.; Costen, M. L.; Bennett, D. I. G.; Kłos, J.; Alexander, M. H.; Dagdigian, P. J., Depolarization of rotational angular momentum in $\mathrm{CN}\left(\mathrm{A}^{2} \Pi, \mathrm{v}=4\right)+\mathrm{Ar}$ collisions. J. Chem. Phys. 2012, 136, 164306.

21. McGurk, S. J.; McKendrick, K. G.; Costen, M. L.; Alexander, M. H.; Dagdigian, P. J., Parity-dependent oscillations in collisional polarization transfer: $\mathrm{CN}\left(\mathrm{A}^{2} \Pi, \mathrm{v}=4\right)+\mathrm{Ar}$. J. Chem. Phys. 2013, 139, 124304.

22. Tully, F. P.; Goldsmith, J. E. M., Kinetic study of the hydroxyl radical - propene reaction. Chem. Phys. Lett. 1985, 116, 345-352.

23. Hause, M. L.; Hall, G. E.; Sears, T. J., Sub-Doppler laser absorption spectroscopy of the $\mathrm{A}^{2} \Pi-\mathrm{X}^{2} \Sigma^{+}(1,0)$ band of $\mathrm{CN}$ : Measurement of the ${ }^{14} \mathrm{~N}$ hyperfine parameters in $\mathrm{A}^{2} \Pi \mathrm{CN} . J$. Mol. Spectrosc. 2009, 253, 122-128.

24. Hause, M. L.; Hall, G. E.; Sears, T. J., Sub-Doppler Stark spectroscopy in the A-X $(1,0)$ band of CN. J. Phys. Chem. A 2009, 113, 13342-13346.

25. Forthomme, D.; McRaven, C. P.; Sears, T. J.; Hall, G. E., Argon-induced pressure broadening, shifting and narrowing in the $\mathrm{CN} \mathrm{A}{ }^{2} \Pi-\mathrm{X}^{2} \Sigma^{+}$(1-0) band. J. Phys. Chem. A 2013, $117,50-55$. 
26. North, S. W.; Marr, A. J.; Furlan, A.; Hall, G. E., Nonintuitive asymmetry in the threebody photodissociation of $\mathrm{CH}_{3}$ COCN. J. Phys. Chem. A 1997, 101 (49), 9224-9232.

27. Halpern, J. B.; Huang, Y.; Titarchuk, T., Radiative and Collisional Processes in CN A ${ }^{2} \Pi_{\mathrm{i}}$. Astrophysics and Space Science 1996, 236, 11-17.

28. Prasad, C. V. V.; Bernath, P. F., Fourier transform jet-emission spectroscopy of the A-X transition of CN. J. Mol. Spectrosc. 1992, 156, 327-340.

29. Katayama, D. H.; Miller, T. A.; Bondybey, V. E., Radiative decay and radiationless deactivation in selectively excited CN. J. Chem. Phys. 1979, 71, 1662-1669.

30. Dagdigian, P. J., State-resolved collision-induced electronic transitions. Annu. Rev. Phys. Chem. 1997, 48, 95-123.

31. Alexander, M. H.; Hall, G. E.; Dagdigian, P. J., The approach to equilibrium: detailed balance and the master equation. J. Chem. Educ. 2011, 88, 1538-1543.

32. Fano, U.; Macek, J. H., Impact excitation and polarization of the emitted light. Rev. Mod. Phys. 1973, 45, 553-573.

33. Greene, C. H.; Zare, R. N., Photofragment alignment and orientation. Annu. Rev. Phys. Chem. 1982, 33, 119-150.

34. North, S. W.; Zheng, X. S.; Fei, R.; Hall, G. E., Line shape analysis of Doppler broadened frequency-modulated line spectra. J. Chem. Phys. 1996, 104, 2129-2135.

35. Bjorklund, G. C., Frequency-modulation spectroscopy: a new method for measuring weak absorptions and dispersions. Opt. Lett. 1980, 5, 15-17.

36. Coy, S. L.; Halle, S. D.; Kinsey, J. L.; Field, R. W., Pressure-induced rotational energy transfer in $\mathrm{H}_{2} \mathrm{CO}$ (A) $v_{4}=1$ : dipolar M-dependence with no single-collision elastic contribution. J. Mol. Spectrosc. 1992, 153, 340-375. 
37. Fei, R.; Lambert, H. M.; Carrington, T.; Filseth, S. V.; Sadowski, C. M., Direct measurement of thermal rate constants for state-to-state rotational energy transfer in collisions of CN (X, v=2, N) with He. J. Chem. Phys. 1993, 100, 1190-1201.

38. Klaassen, J. J.; Coy, S. L.; Steinfeld, J. I.; Abel, B., Direct measurement of rotational and vibrational relaxation in methane overtone levels by time-resolved infrared double-resonance spectroscopy. J. Chem. Phys. 1994, 101, 10533-10547.

39. Olkhov, R. V.; Smith, I. W. M., Rate coefficients for reaction and for rotational energy transfer in collisions between $\mathrm{CN}$ in selected rotational levels $(\mathrm{X}, \mathrm{v}=2, \mathrm{~N}=0,1,6,10,15$, and 20) and $\mathrm{C}_{2} \mathrm{H}_{2}$. J. Chem. Phys, 2007, 126, 134314.

40. Olkhov, R. V.; Smith, I. W. M., Energy transfer in thermal and hyperthermal collisions between $\mathrm{CN}(\mathrm{X}, \mathrm{v}=2)$ in selected rotational levels $(\mathrm{Ni}=0,1,6,10,15$ and 20) and $\mathrm{N} 2$. Phys. Chem. Chem. Phys. 2006, 8, 5643-5652.

41. Kabir, M. H.; Antonov, I. O.; Merritt, J. M.; Heaven, M. C., Experimental and theoretical investigations of rotational energy transfer in $\mathrm{HBr}+\mathrm{He}$ collisions. J. Phys. Chem. A 2010, 114, 11109-11116.

42. Hall, G. E.; North, S. W., Transient laser frequency modulation spectroscopy. Annu. Rev. Phys. Chem. 2000, 51, 243-274.

43. Fei, R.; Adelman, D. E.; Carrington, T.; Dugan, C. H.; Filseth, S. V., Rotational energy transfer in collisions between $\mathrm{CN}(\mathrm{X}, \mathrm{v}=2)$ and argon. Comparison with results for helium. Chem. Phys. Lett. 1995, 232, 547-553.

44. Stewart, B. A.; Stephens, T. N.; Lawrence, B. A.; McBane, G. C., Rovibrational Energy Transfer in Ne-Li $2(\mathrm{~A}, \mathrm{v}=0)$ : Comparison of Experimental Data and Results from Classical and Quantum Calculations. J. Phys. Chem. A 2010, 114, 9875-9885. 
45. Lique, F.; Spielfiedel, A.; Feautrier, N.; Schneider, I. F.; Klos, J.; Alexander, M. H., Rotational excitation of $\mathrm{CN}(\mathrm{X})$ by He: theory and comparison with experiments. J. Chem. Phys. 2010, $132,024303$.

46. Guo, J.; Sadowski, C. M.; Gao, Q.; Morgan, F. J., State-to-state and total rotational energy transfer rate constants for $\mathrm{CN}\left(\mathrm{B}^{2} \Sigma^{+}, \mathrm{v}=0, \mathrm{~N}\right)+\mathrm{He}$ and $\mathrm{C}_{2} \mathrm{~N}_{2}$. J. Chem. Phys. 2000, 113, 7276-7285.

47. Hay, S.; Shokoohi, F.; Callister, S.; Wittig, C., Collisional metastability of high rotational states of CN (X $\left.{ }^{2} \Sigma^{+}, \mathrm{v}^{\prime \prime}=0\right)$. Chem. Phys. Lett. 1985, 118, 6-11.

48. Roueff, E.; Lique, F., Molecular excitation in the interstellar medium: recent advances in collisional, radiative and chemical processes. Chem. Rev. 2013, 113, 8906-8938.

49. Bruggeman, P. J.; Sadeghi, N.; Schram, D. C.; Linss, V., Gas temperature determination from rotational lines in non-equilibrium plasmas: a review. Plasma Sources Sci. Technol. 2014, $23,023001$.

50. Polanyi, J. C.; Woodall, K. B., Mechanism of rotational relaxation. J. Chem. Phys, 1972, $56,1563-1572$.

51. Brunner, T. A.; Smith, N.; Karp, A. W.; Pritchard, D. E., Rotational energy transfer in $\mathrm{Na}_{2}{ }^{*}$ (A) colliding with $\mathrm{Xe}, \mathrm{Kr}, \mathrm{Ar}, \mathrm{He}, \mathrm{H}_{2}, \mathrm{CH}_{4}$ and $\mathrm{N}_{2}$. J. Chem. Phys, 1981, 74, 3324-3341.

52. Ramaswamy, R.; Depristo, A. E.; Rabitz, H., On the correlation of rotationally inelastic rates: a scaling theoretical analysis. Chem. Phys. Lett. 1979, 61, 495-498.

53. Pattengill, M. D., Rotational Excitation III: Classical Trajectory Methods. In AtomMolecule Collision Theory, Bernstein, R. B., Ed. Plenum Press: New York, 1979; pp 359-375. 
54. Aoiz, F. J.; Brouard, M.; Eyles, C. J.; Kłos, J.; de Miranda, M. P., The collisional depolarization of $2 \mathrm{~S}+1 \Sigma$ radicals by closed shell atoms: Theory and application to $\mathrm{OH}\left(\mathrm{A}^{2} \Sigma^{+}\right)+$ Ar. J. Chem. Phys. 2009, 130, 044305.

55. Brouard, M.; Bryant, A.; Chang, Y.-P.; Cireasa, R.; Eyles, C. J.; Green, A. M.; Marinakis, S.; Aoiz, F. J.; Kłos, J., Collisional depolarization of OH(A) with Ar: Experiment and theory. J. Chem. Phys. 2009, 130, 044306.

56. LaBudde, R. A.; Bernstein, R. B., Classical study of rotational excitation of a rigid rotor: $\mathrm{Li}^{+}+\mathrm{H}_{2}$. J. Chem. Phys. 1971, 55, 5499-5516.

57. Clegg, S. M.; Parmenter, C. S., Inelastic scattering from glyoxal: collisional kinematics rather than the interaction potential dominates rotational channel selection. J. Chem. Phys, 2006, $125,133110$.

58. HIBRIDON, a package of programs for the time-independent quantum treatment of inelastic collisions and photodissociation written by M. H. Alexander, D. E. Manolopoulos, H.-J. Werner, and B. Follmeg, P. Dagdigian, Q. Ma, and others. More information and/or a copy of the code can be obtained from the website http://www2.chem.umd.edu/groups/alexander/hibridon. 


\section{Figure Captions}

Figure 1. Experimental block diagram for FM-detected saturation recovery kinetics. cw probe: Ar+ pumped Ti:sapphire laser; ns bleach: Nd:YAG-pumped dye laser; EOM: electro-optic phase modulator; PEM: photoelastic polarization modulator; DSO: digital storage oscilloscope; $\varphi$ shift: voltage controlled phase-shifter.

Figure 2. Typical transient FM signal with saturation recovery kinetics. Signal is observed at $10895.484 \mathrm{~cm}^{-1}$, near the peak of the FM signal for the $\mathrm{CN} \mathrm{P}_{2}(6)$ line in the (1-0) band for a sample of 10 mTorr $\mathrm{CH}_{3} \mathrm{COCN}$ and $590 \mathrm{mT}$ Ar. Photolysis laser fires $12 \mu \mathrm{s}$ before $t=0$, when the bleach laser fires. The bleach laser is tuned to the center of the $\mathrm{P}_{2}(6)$ line in the (4-0) band at $16103.64 \mathrm{~cm}^{-1}$. Inset: expanded saturation transient, expressed as fractional depletion vs. time for a single polarization. Solid line fit: single exponential with final offset.

Figure 3. Thermal fits to FM depletion signals at selected times following bleach laser pulse. Symbols depict transient FM depletion data, polarization averaged, and optimally rotated to isolate absorption- and dispersion-phase spectra. Solid lines illustrate best-fit absorption $\left(A_{F M}\right)$ and dispersion $\left(D_{F M}\right)$ thermal lineshapes, with an adjustable temperature, Doppler-integrated intensity, and phase angle for each time slice. This example is for a sample containing 10 mTorr $\mathrm{CH}_{3} \mathrm{COCN}$ and 590 mTorr Ar, probed and bleached on a $\mathrm{R}_{1}(0)$ line.

Figure 4. Time slice fits to total depletion and translational temperature for the same sample illustrated in Figure 3. a) translational temperature vs. time after saturation, showing rapid cooling and slow reheating; b) Doppler-integrated depletion amplitude, normalized to initial value; c) semi-logarithmic plot of the same data shown in b). 
Figure 5. Variation in the observed decay kinetics at selected Doppler detunings for the $\mathrm{P}_{2}(4)$ line, with 190 mTorr Ar added to 10 mTorr $\mathrm{CH}_{3} \mathrm{COCN}$. The full frequency-dependent decay was treated with a global fit; FM decay curves are shown here at Doppler detunings of $\mathrm{x}_{\mathrm{D}}=$ \pm 0.65 (average signals near the + and - peak FM signals, about $\pm 0.010 \mathrm{~cm}^{-1}$ from line center) and $\mathrm{x}_{\mathrm{D}}= \pm 1.5$ (average of signals about $\pm 0.024 \mathrm{~cm}^{-1}$ from line center, where the FM signal intensity has dropped to about one half of the maximum). Solid lines are the corresponding frequency-selected decay kinetics from the global fit, normalized to the same initial depletion. Measured decay signals (symbols) have been scaled by the same normalization factors as the fits.

Figure 6. Pressure dependence of initial recovery rates for selected rotational states in $\mathrm{He}$ and Ar. The $\mathrm{CH}_{3} \mathrm{COCN}$ precursor partial pressure was maintained at 10 mTorr, contributing a strong rotational state dependence to the intercepts.

Figure 7. Total RET rate constants for $\mathrm{CN}\left(X^{2} \Sigma^{+}\right)+$He vs. initial rotational state. Open symbols are $295 \mathrm{~K}$ thermal rate constants, summed over all inelastic channels from the Hibridon scattering calculations of Lique, et al. ${ }^{45}$ Blue squares are the SEP/LIF total removal rate constants from Fei et al. ${ }^{37}$ converted from (ns· Torr) ${ }^{-1}$ units, assuming $300 \mathrm{~K}$ ideal gas density. Red triangles labeled FM are this work, based on the global fits described in the text. Points and connecting lines are from quasi-classical trajectory calculations for total inelastic rate constants, (this work).

Figure 8. Total RET rate constants for $\mathrm{CN}\left(X^{2} \Sigma^{+}\right)+\operatorname{Ar}$ vs. initial rotational state. Open symbols are $298 \mathrm{~K}$ thermal rate constants, summed over all inelastic channels from the Hibridon scattering calculations (this work). Blue squares are the SEP/LIF total removal rate constants from Fei et al. $^{43}$ converted from (ns. Torr) ${ }^{-1}$ units, assuming $300 \mathrm{~K}$ ideal gas density. Red 
triangles labeled FM are this work, based on the global fits described in the text. Points and connecting lines are from quasi-classical trajectory calculations for total inelastic rate constants (this work).

Figure 9. (a) Speed-dependent rotationally inelastic cross sections for $\mathrm{Ar}+\mathbf{C N}\left(X^{2} \Sigma^{+}\right)$in selected initial rotational states, from quantum scattering calculations. (b) Doppler-resolved total inelastic rate for selected initial rotational states, using Eq. 10 to average $v_{r} \sigma\left(v_{r}\right)$ over $f\left(v_{r} \mid v_{z}\right)$, the conditional relative velocity given the Doppler shift. Solid lines are the numerical non-thermal averages. Dashed lines are best fits to empirical form $k\left(x_{D}\right)=k_{0} \sqrt{1+b x_{D}}$. 


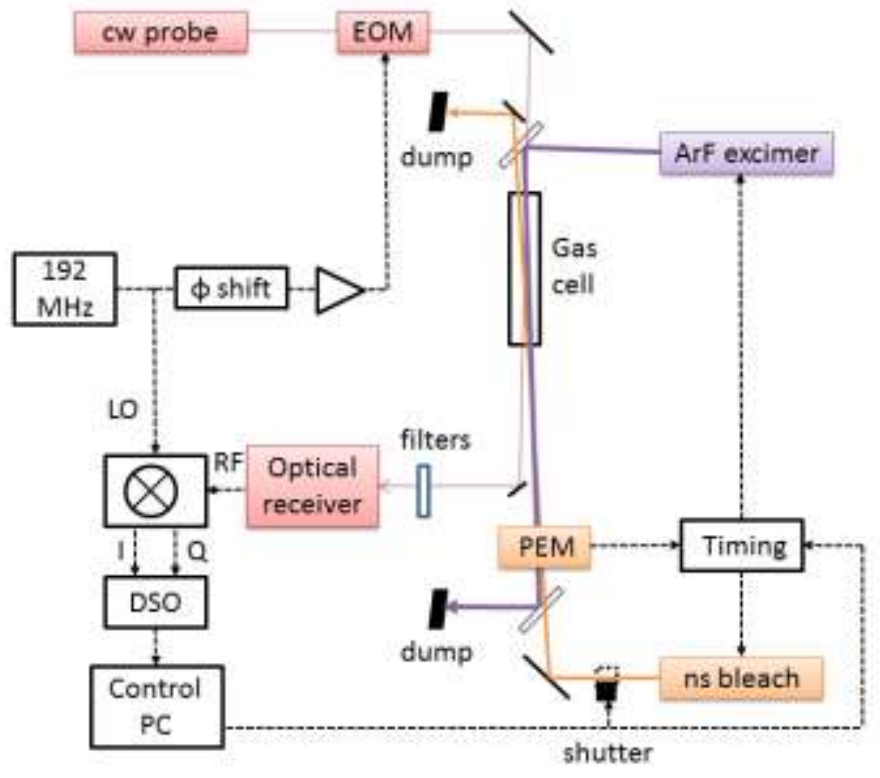

Figure 1 


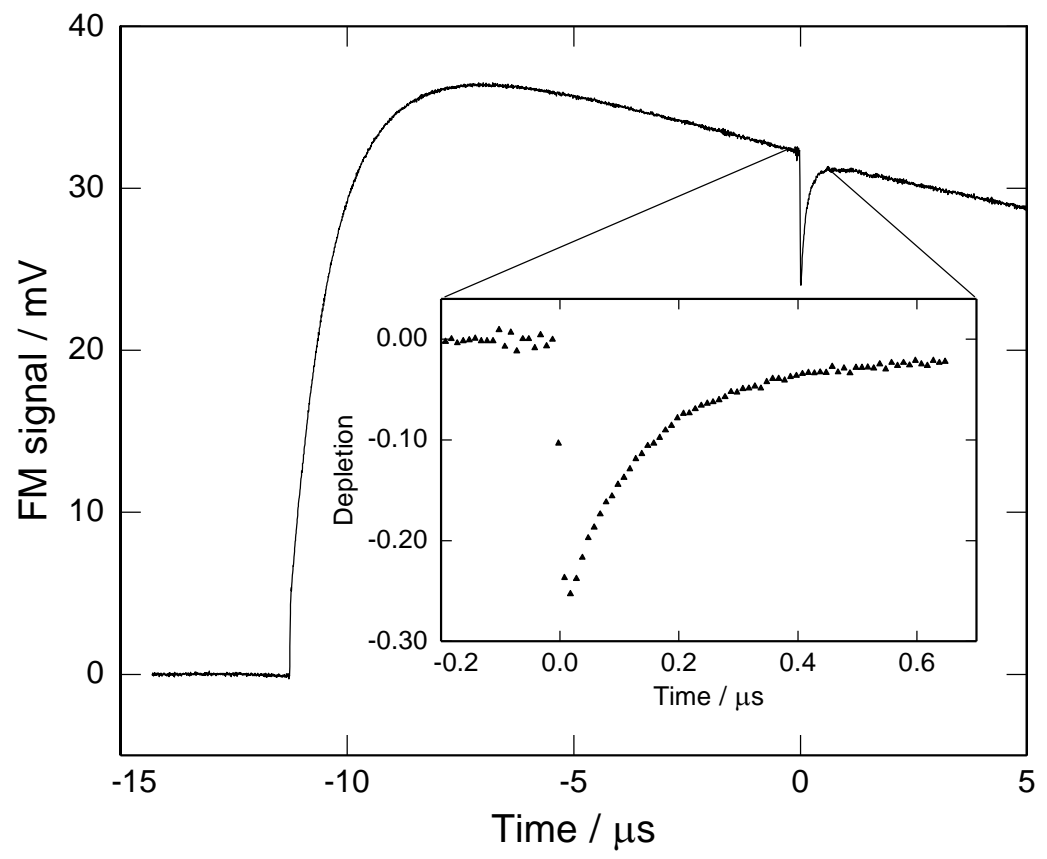

Figure 2 

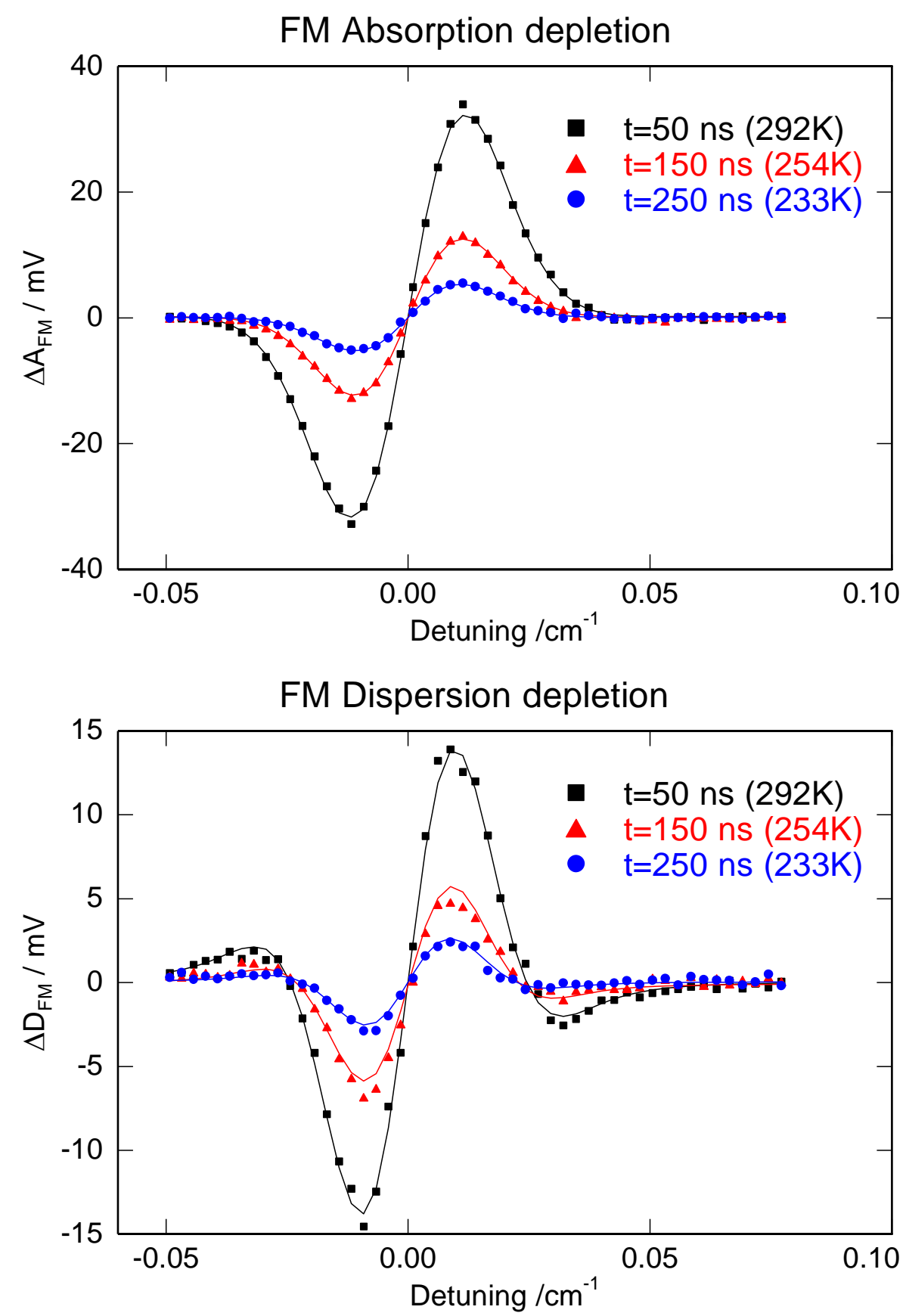

Figure 3 

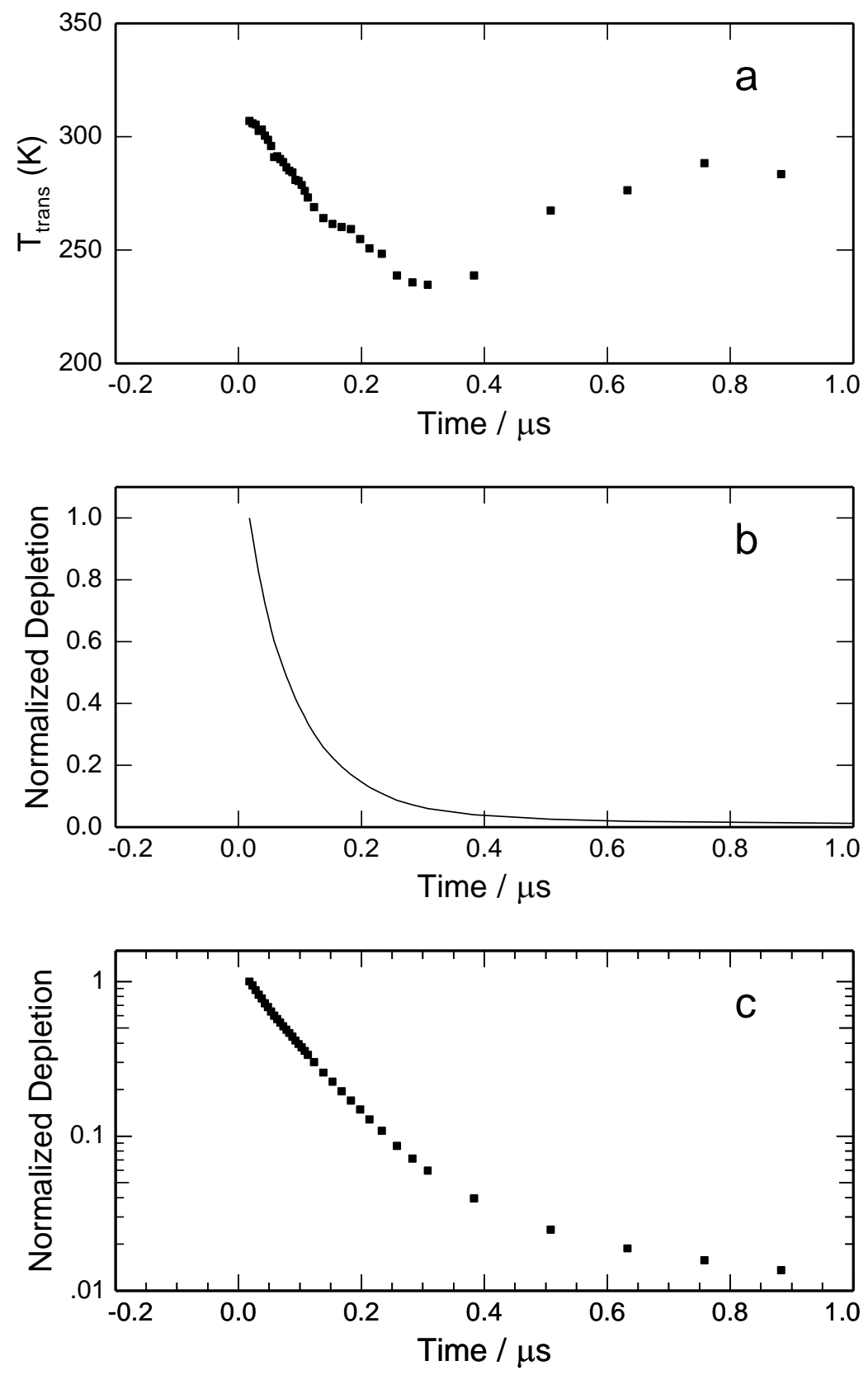

Figure 4 


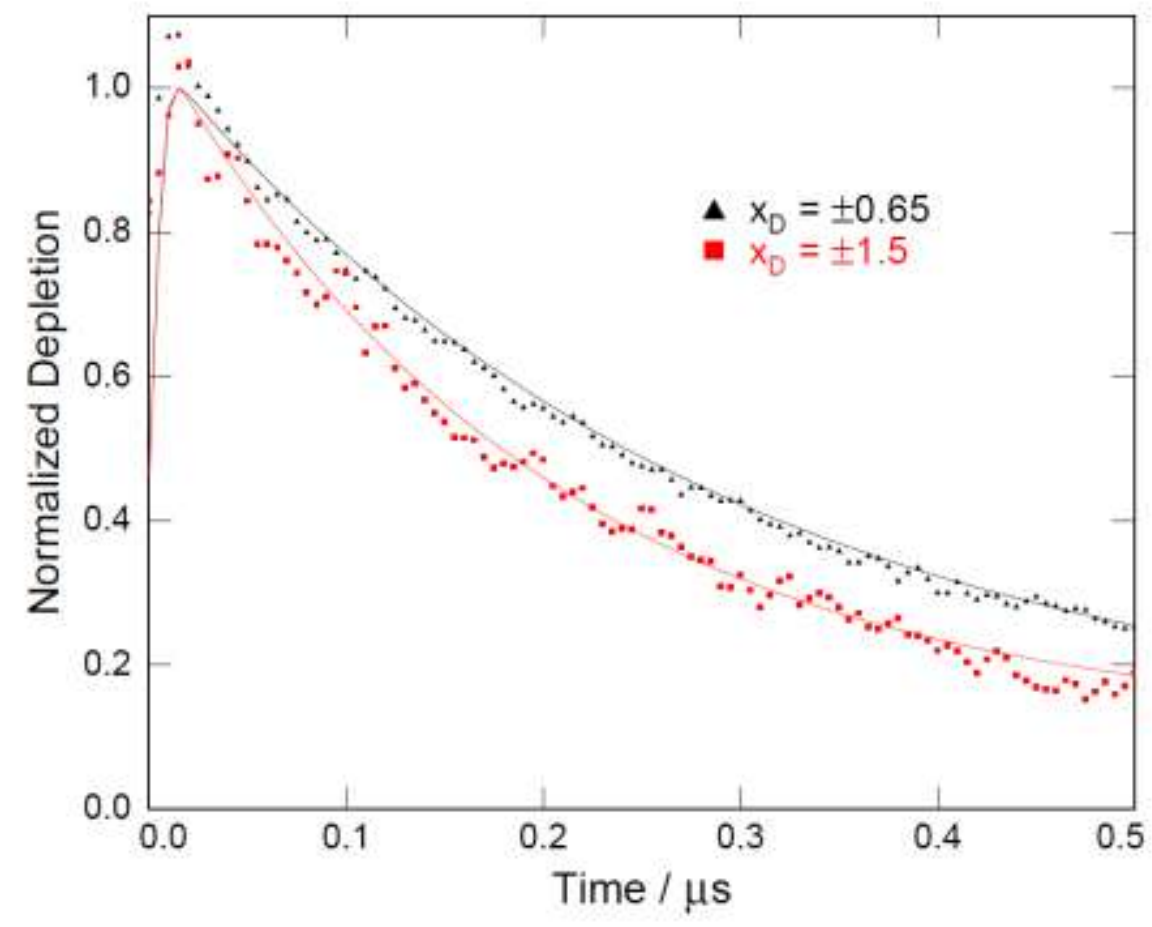

Figure 5 


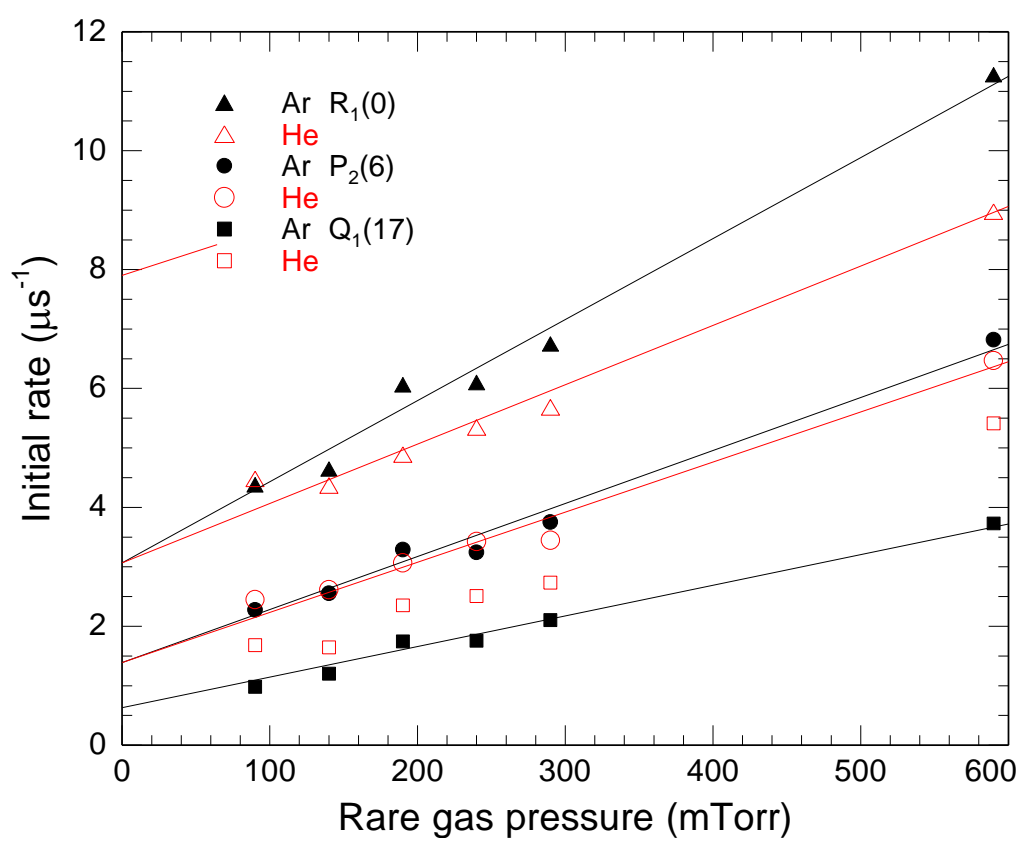

Figure 6 


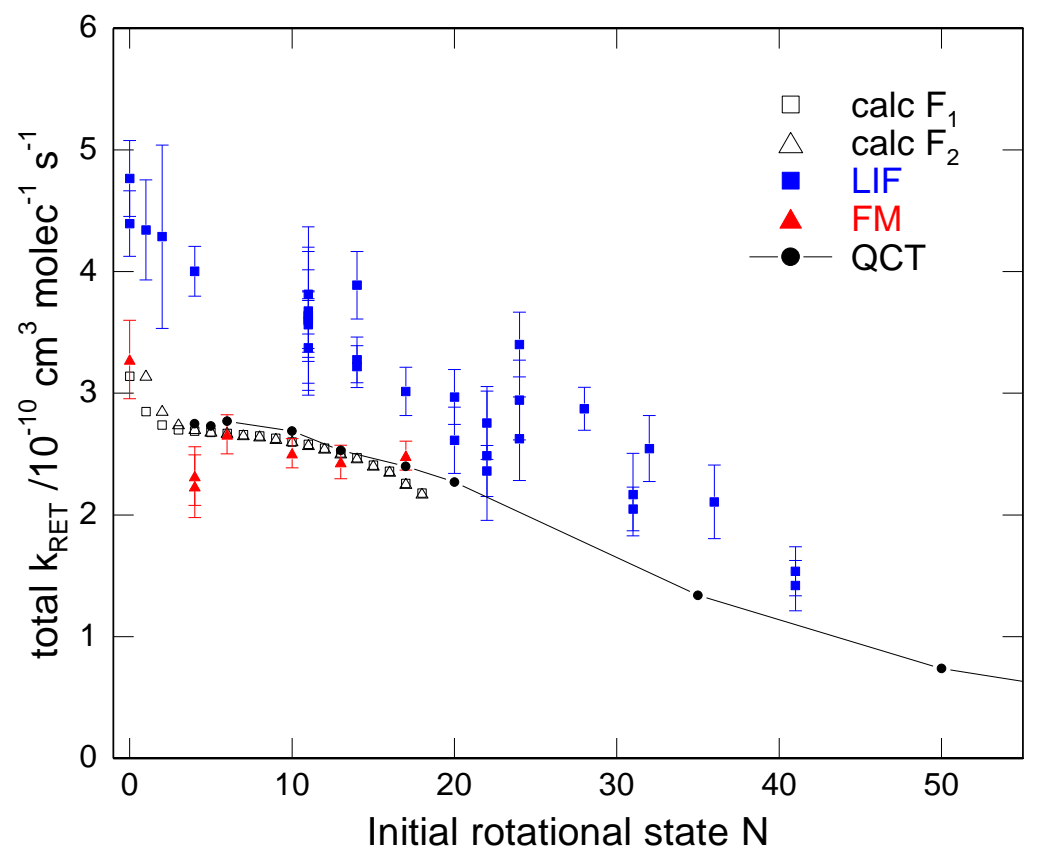

Figure 7 


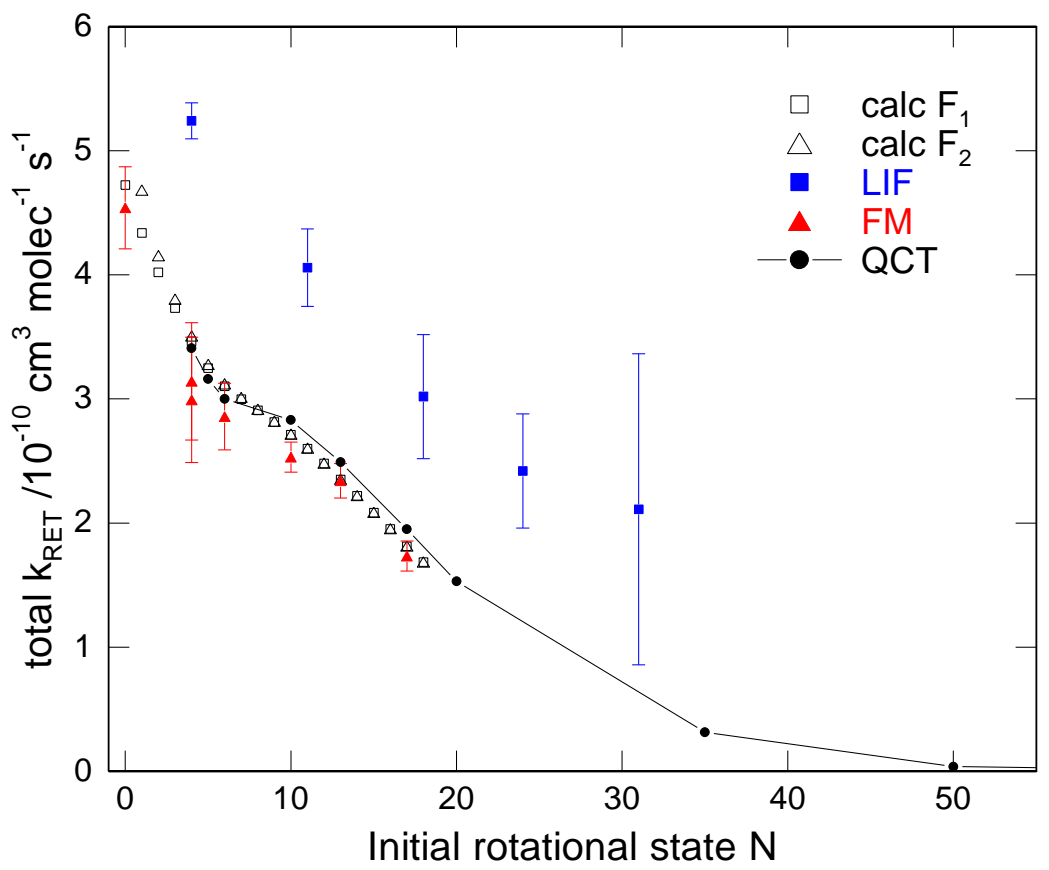

Figure 8 

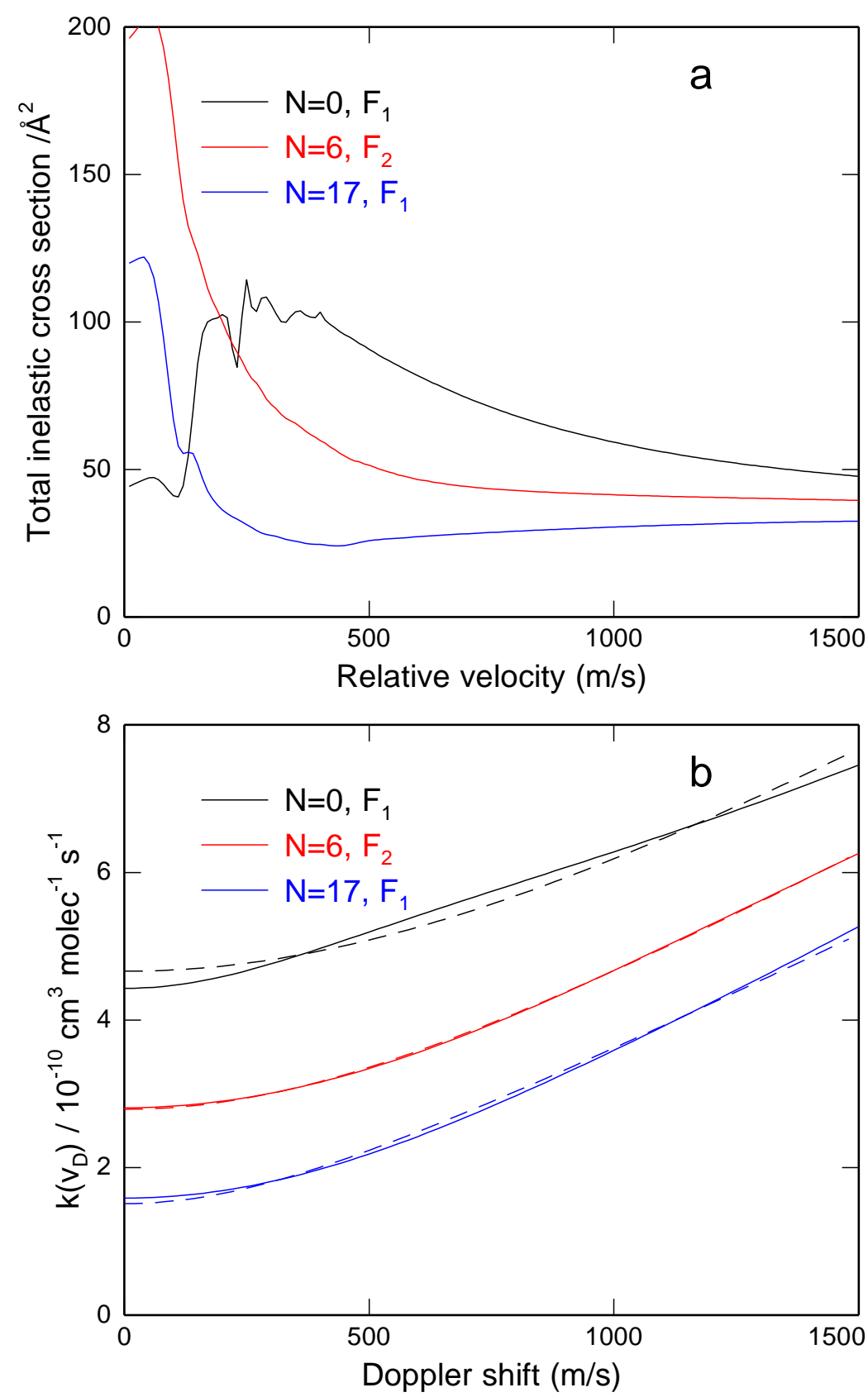

Figure 9 


\section{TOC-Graphic}

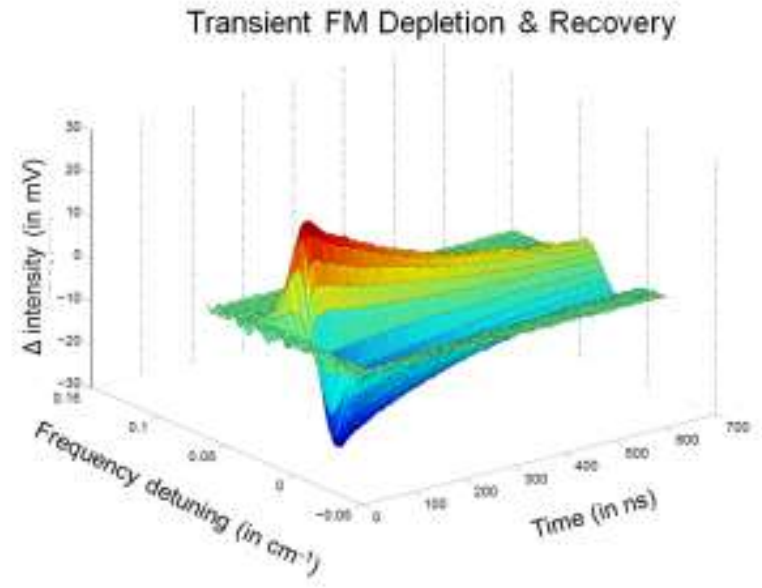




\title{
Supplemental Information: Doppler-Resolved Kinetics of Saturation Recovery
}

\author{
Damien Forthomme, Michael L. Hause, Hua-Gen Yu, Paul J. Dagdigian \\ Trevor J. Sears, and Gregory E. Hall
}

\section{Supplement 1. Temperature dependence of FM line shapes}

Frequency modulated line shapes of thermal, Doppler-broadened lines are more sensitive to the temperature than are direct absorption line shapes. A simple example illustrates this. Consider a Doppler broadened Gaussian absorption line at a reference temperature of $300 \mathrm{~K}$, with a unit maximum intensity, plotted on a normalized frequency axis, $x_{D}=\left(\omega-\omega_{0}\right) / \omega_{D}$, where $\omega_{D}=\omega_{0} \sqrt{2 k_{B} T / m} / c$ is the Doppler shift at the most probable velocity:

$$
A\left(x_{D}\right)=e^{-x_{D}^{2}} .
$$

Cooling to $250 \mathrm{~K}$ with no change in density will increase the peak amplitude and decrease

the line width by a factor of $\sqrt{300 / 250}$, when plotted against the same $300 \mathrm{~K}$ normalized frequency, as shown in Fig S1a.

For a RF modulation frequency 0.4 times the Doppler shift of the most likely thermal velocity at $300 \mathrm{~K},\left(x_{R F}=0.4\right)$, typical of our current experiments, the corresponding FM line shape in the absorption phase will be a difference of frequency offset Gaussians, as shown in Fig S1b according to Equation S2 (neglecting the effects of second-order sidebands):

$$
A_{F M}\left(x_{D}\right)=A\left(x_{D}+x_{R F}\right)-A\left(x_{D}-x_{R F}\right) \text {. }
$$



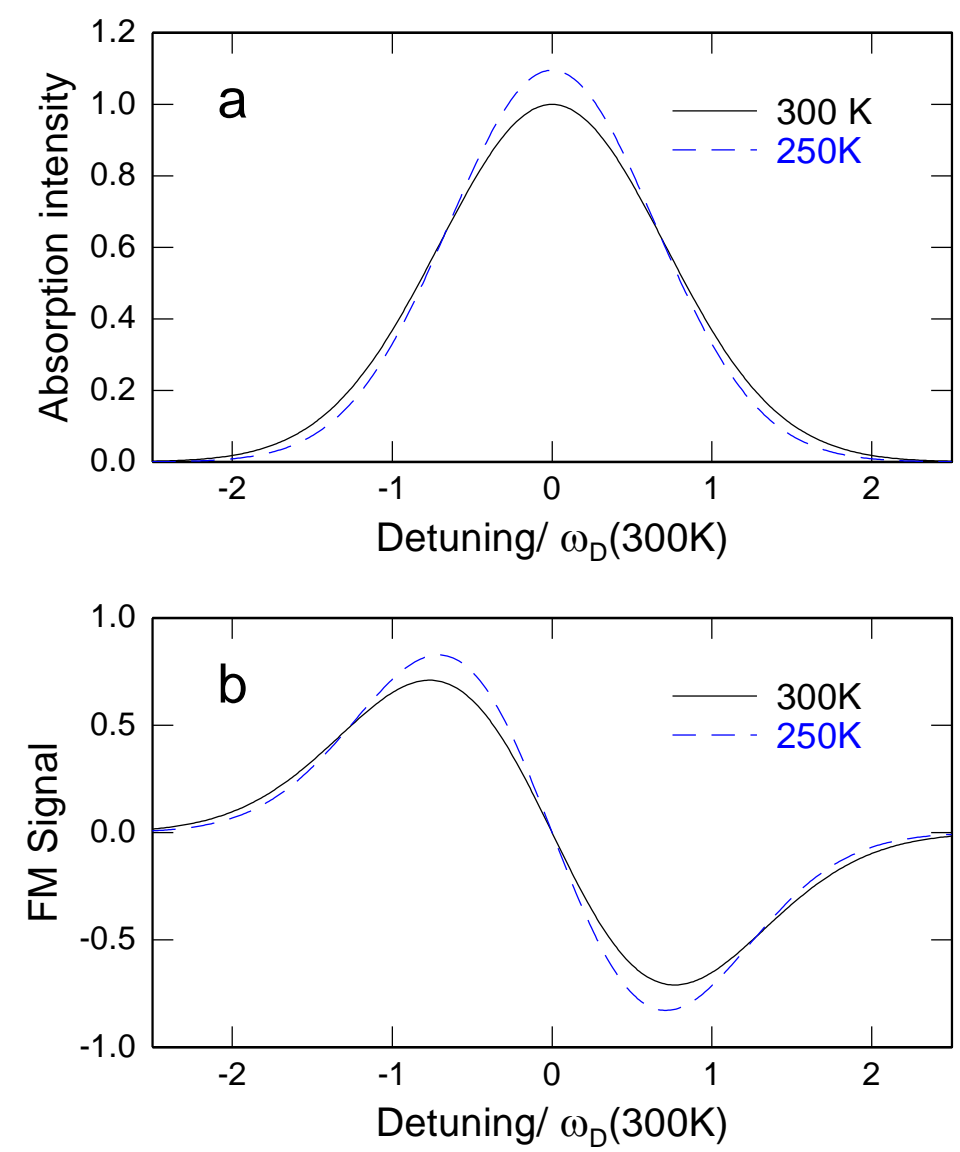

Figure S1. Normalized direct absorption (a) and absorption phase FM signals (b) for a fixed total population at $300 \mathrm{~K}$ and $250 \mathrm{~K}$. A modulation frequency 0.4 times the Doppler shift at the most likely velocity is used for this example.

The intensity of the colder FM spectrum exceeds that of the warmer sample over a wider range of detunings, and by a larger factor, compared to direct absorption. Figure S2 shows the ratio of signal intensity at $250 \mathrm{~K}$ relative to $300 \mathrm{~K}$ as a function of Doppler detuning for these example direct absorption and FM line shapes. 


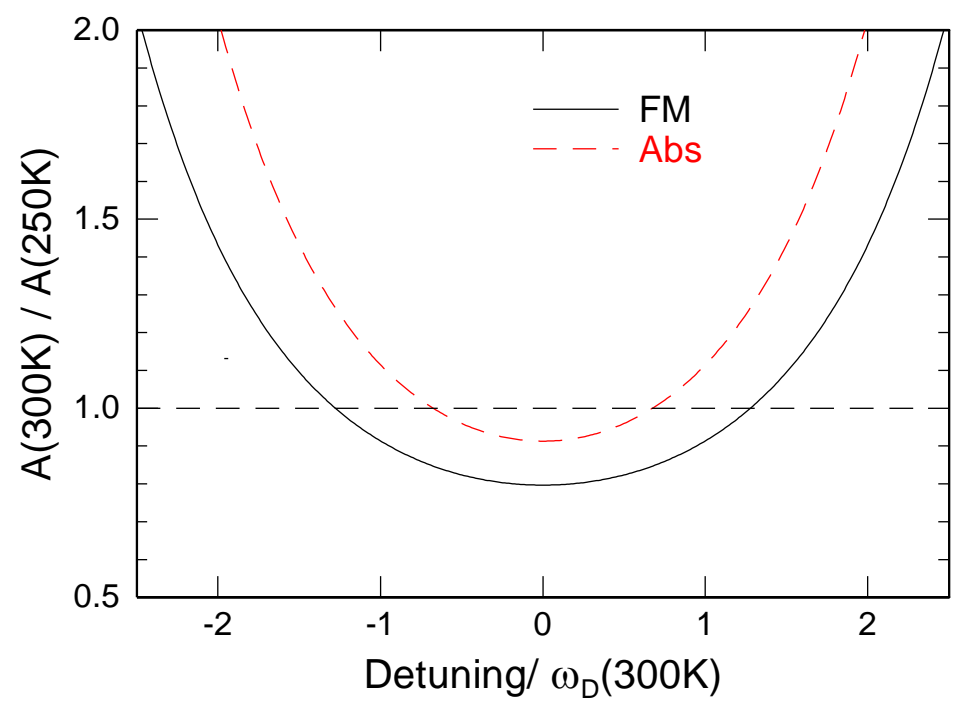

Figure S2. Relative intensity of $300 \mathrm{~K}$ vs. $250 \mathrm{~K}$ signals as a function of detuning for direct absorption and absorption phase FM monitoring.

The warning to be drawn from this illustration is that when monitoring a population by narrow-band direct absorption (or LIF) measurements, if the temperature cools from $300 \mathrm{~K}$ to $250 \mathrm{~K}$ during the first decay lifetime, 1/k, of a single exponential decay, the initial apparent decay rate will be reduced by about $9 \%$ at line center, and be approximately undistorted at a detuning of $x_{D}= \pm 0.68$, near the detuning of half-maximum. At larger Doppler shifts, the apparent decay rate will exceed the thermally averaged decay rate by an increasing margin. Analyzing the decay rate of the Doppler integrated area rather than the decay at line center is a common and sensible treatment for such heterogeneous speed-dependent kinetics.

For FM spectroscopy, the trend is the same, but the discrepancy is larger. Near line center (where the FM signal vanishes) the apparent decay rate will be reduced by more than $20 \%$, and it will remain slower than the thermally averaged decay rate at all detunings $\left|x_{D}\right| \leq 1.28$. When the speed distribution is changing along with the total population, kinetic measurements at fixed frequency can be subject to systematic error, and an analysis that explicitly considers the time- 
dependent line shape is required for accurate kinetic interpretation, as has been implemented in sections 3.2 and 3.3 of the main paper.

\section{Supplement 2. Quantum Scattering Calculations of Rotationally Inelastic Collisions of $\mathrm{CN}(X)$ by Rare Gases}

The HIBRIDON suite of programs ${ }^{1}$ was employed to carry out close-coupling calculations of state-to-state integral cross sections vs. collision energy for collision of $\mathrm{CN}(X)$ with argon. The $\mathrm{CN}(X, v=0)-\mathrm{Ar}$ interaction potential, taken from ref 2 , was parameterized as a table of Legendre expansion coefficients $V_{\lambda}(R)$ :

$$
V(R, \theta)=\sum_{\lambda=0}^{10} V_{\lambda} P_{\lambda}(\cos \theta),
$$

where the $\mathrm{CN}$ bond length is fixed at the $v=0$ average value, $R$ and $\theta$ are the other two Jacobi coordinates and $P_{\lambda}(\cos \theta)$ is a Legendre polynomial. The angular expansion coefficients $V$ on a grid of $R$ values can be generated from routines and data files included in the current download of HIBRIDON. ${ }^{1}$

Convergence of the cross sections was checked with respect to the size of the rotational basis and the number of partial waves, and these varied as a function of the total energy. At the

highest energy considered $\left(2000 \mathrm{~cm}^{-1}\right)$, the rotational basis included all levels with $j \leq 22.5$, and the scattering calculations included all total angular momenta $J \leq 500.5$.

Total removal cross sections for a given rotational level as a function of collision energy were determined by summing all the state-to-state integral cross sections for transitions out of that level. For the calculation of thermal rate constants, the cross sections were computed on a grid of collision energies, up to a total energy of $2000 \mathrm{~cm}^{-1}$ and were averaged over a roomtemperature $(298 \mathrm{~K})$ Maxwellian distribution of relative velocities. ${ }^{3}$ 


\section{Supplement 3. Quasi-Classical Trajectory calculations of rotationally inelastic scattering in $\mathrm{CN}(\mathrm{X})$ by rare gases.}

Classical equations of motion were integrated to investigate the rotationally inelastic scattering of a rigid $\mathrm{CN}$ molecule by a rare gas atom, following standard procedures. ${ }^{4,5,6}$ The diatomic bond length was constrained to be rigid by the method of Lagrange. ${ }^{4}$ The $\mathrm{CN}(X, \mathrm{v}=0)-\mathrm{Ar}$ interaction potential $^{2}$ was the same as was used in the quantum scattering calculations of this work, and the $\mathrm{CN}(X, \mathrm{v}=0)-\mathrm{He}$ potential ${ }^{7}$ was in the same form of an R-dependent Legendre expansion. Both potentials can also be generated using routines and data files included in the current download of Hibridon. ${ }^{1}$ The initial angular momentum of the $\mathrm{CN}$ rotor was fixed for each set of trajectories, while the plane of rotation and initial rotational phase were chosen at random, relative to the initial atomic velocity, while the initial impact parameter, $b$, was set to $b_{\max }$ times the square root of a random uniform number between 0 and 1 . The maximum impact parameter, $b_{\max }$, was chosen large enough to include all rotationally inelastic collisions, based on a small preliminary set of calculations for each initial rotational state and collision partner. The relative velocities were sampled from a Maxwell-Boltzmann speed distribution at $298 \mathrm{~K}$. A split step Verlet integrator was used, ${ }^{8}$ with a full time step of $\sim 0.2 \mathrm{fs}$, typically conserving total energy within $0.1 \%$. Trajectories were started at a separation of $20 \mathrm{a}_{0}$ and terminated when the separation exceeded $20 \mathrm{a}_{0}$. Batches of 50,000 to 500,000 trajectories were run for each initial rotational state investigated. If the angular momentum is conventionally ${ }^{5}$ binned by rounding the final classical angular momentum into the nearest integer multiple of $\hbar$, the total rotationally inelastic rate constants are found to exceed the quantum mechanical scattering results and the experimental values by about $30 \%$, while very accurately matching the shape of the rotational 
state dependence computed by quantum scattering calculations on the same potential. If instead, the threshold for classifying a collision as rotationally inelastic is assigned as a change of at least $\pm \hbar$, the computed total inelastic cross sections and rate constants are smaller and in excellent agreement with both quantum scattering and experimental values where they are available. Figures 6 and 7 in the main paper includes these QCT calculated rate constants for an extended range of rotational states, along with experimental and quantum calculations at lower rotational states. This method can then be considered a way of extrapolating the inelastic rate constants to higher initial rotational states, where the quantum scattering calculations become increasingly demanding, and the classical results should be reliable.

To investigate the relative effects of the potential and the mass of the collider, a series of artificial combinations of potential and mass were compared, using the Ar and He potentials in combination with the mass of $\mathrm{He}, \mathrm{Ne}$ and Ar. Figure S3 summarizes these results, collecting the three different masses for fixed potential in each panel of the plot. It can be seen that changing the potential from He to Ar at fixed mass approximately doubles the rate, without changing the shape of the rotational state dependence significantly. Increasing the mass leads to a steeper reduction in the total inelastic rates from higher initial rotational states regardless of the choice of potential. In this comparison of two generally quite similar potentials, the mass effect is clearly dominant. 
$\mathrm{CN}(\mathrm{X})+\mathrm{M}$ in Ar potential

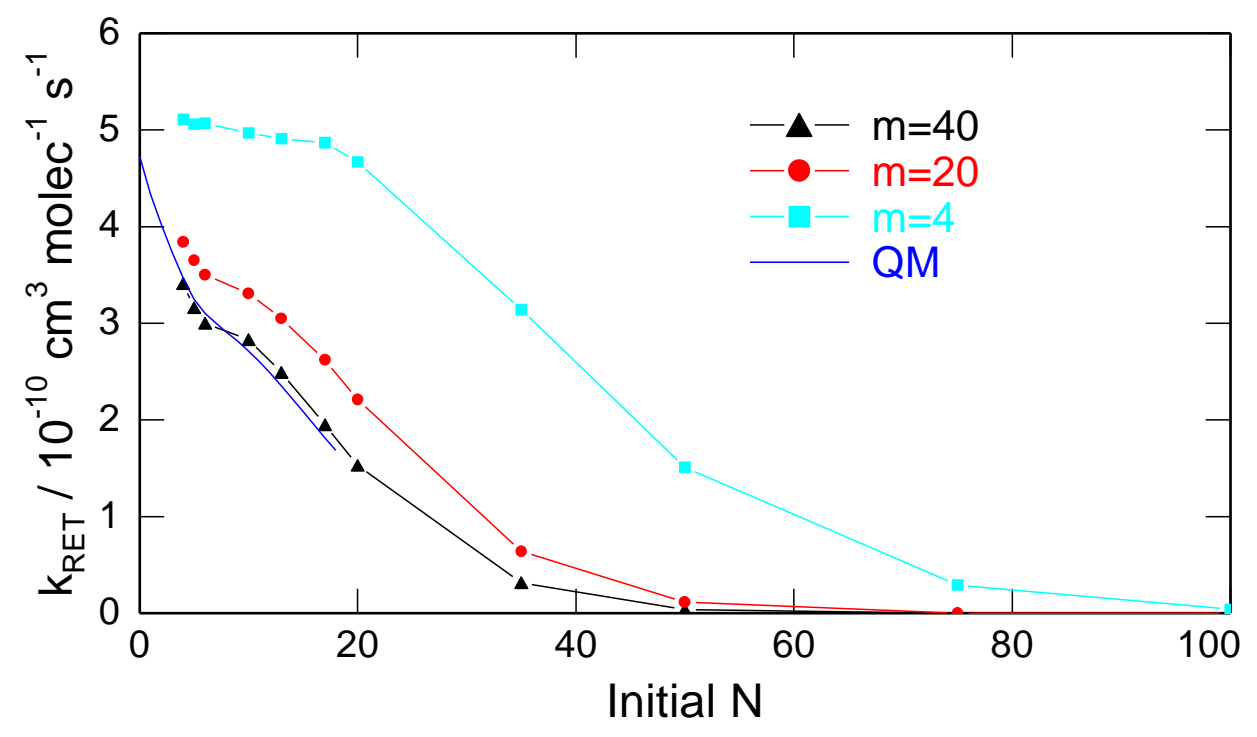

$\mathrm{CN}(\mathrm{X})+\mathrm{M}$ in He potential

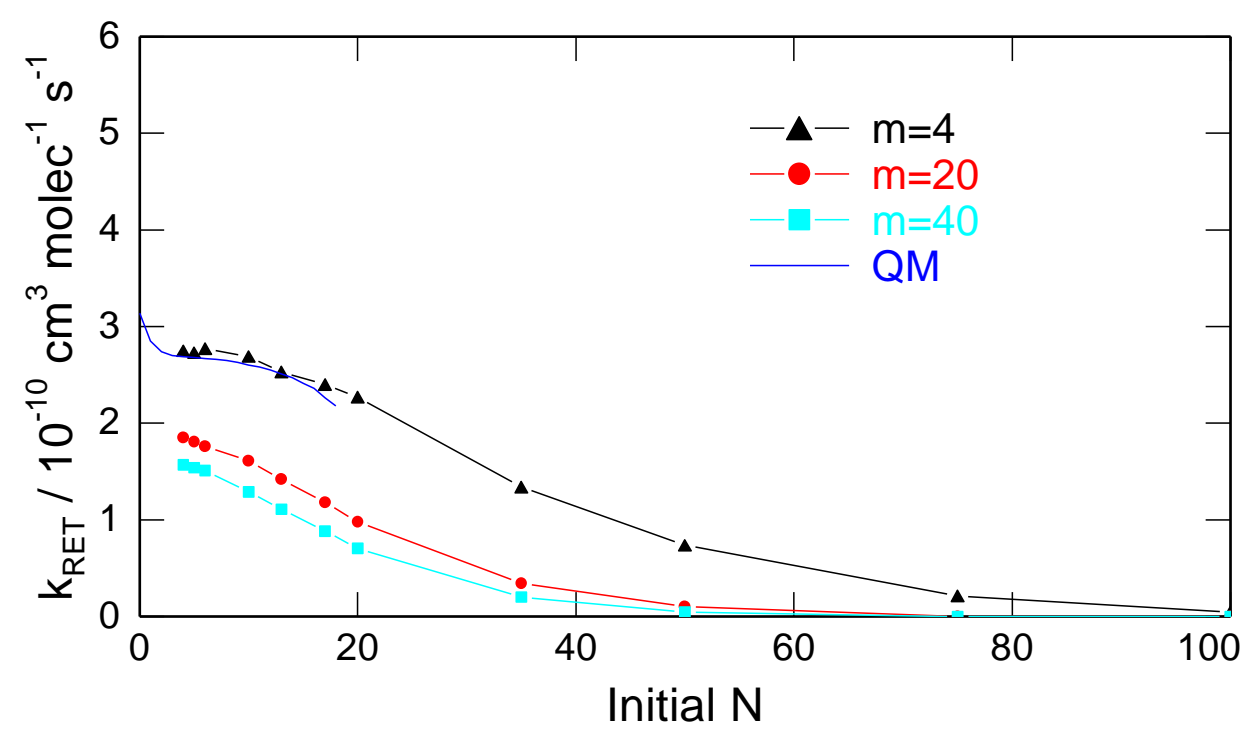

Figure S3. Calculated rotational state dependence of thermal rate constants for total rotationally inelastic collisions of $\mathrm{CN}\left(X^{2} \Sigma^{+}, v=0\right)$ for artificially modified atomic mass of the collider M, using QCT. Masses $\mathrm{m}=4,20,40$ chosen to represent $\mathrm{He}, \mathrm{Ne}$ and Ar. Top panel: calculations using the $\mathrm{CN}+\mathrm{Ar}$ potential; Bottom panel: calculations using $\mathrm{CN}+\mathrm{He}$ potential. QM: blue lines represent corresponding quantum mechanical scattering calculations for He from Lique et al. ${ }^{7}$ and for Ar from this work. 


\section{S4. Supplemental Figures}

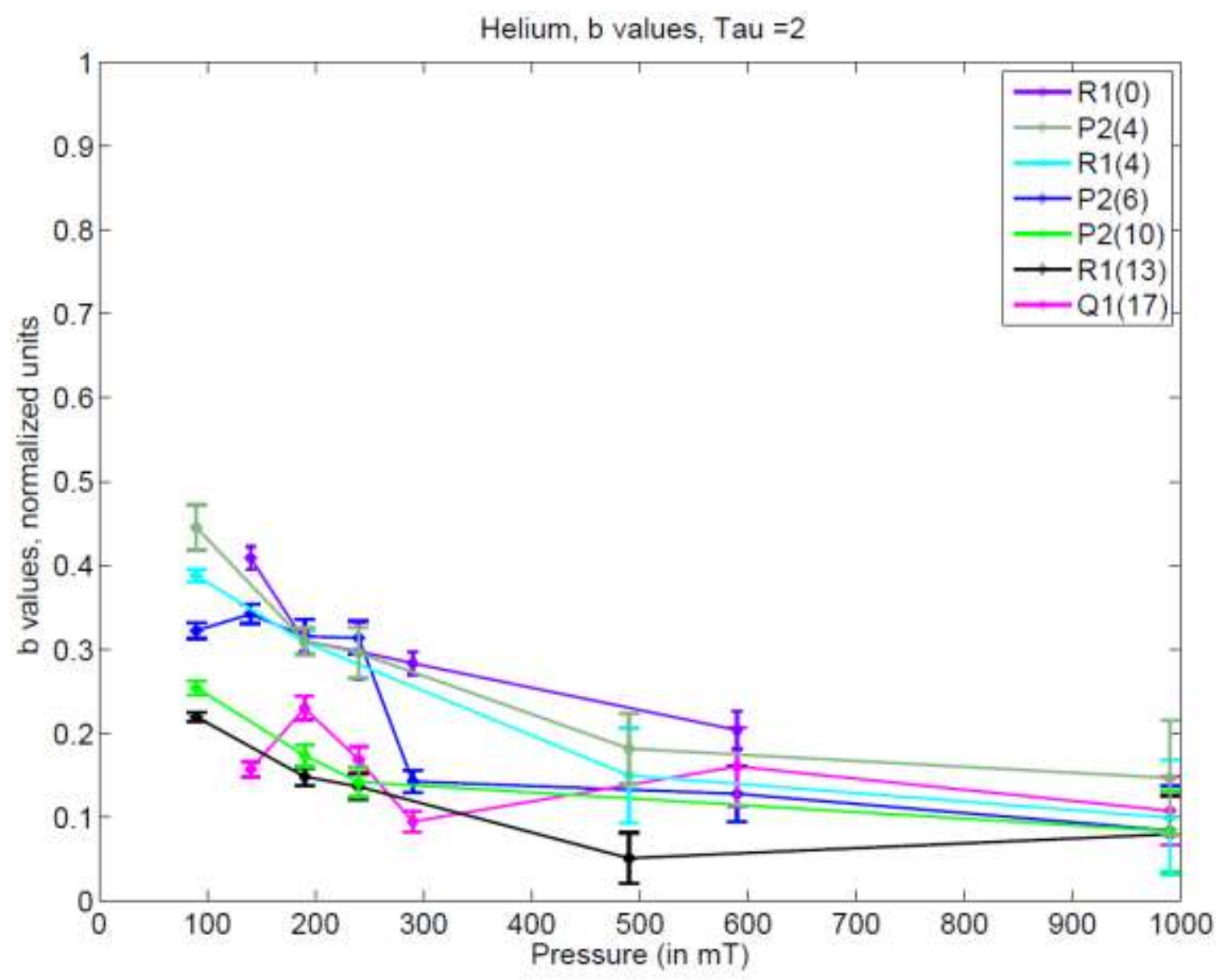

Figure S4. Helium pressure-dependent speed-dependent parameter $b$ for a selection of CN rotational states; $\mathrm{CH}_{3} \mathrm{COCN}$ partial pressure is $10 \mathrm{mTorr}$ for all samples, and contributes disproportionately to the speed dependence at low total pressures. 


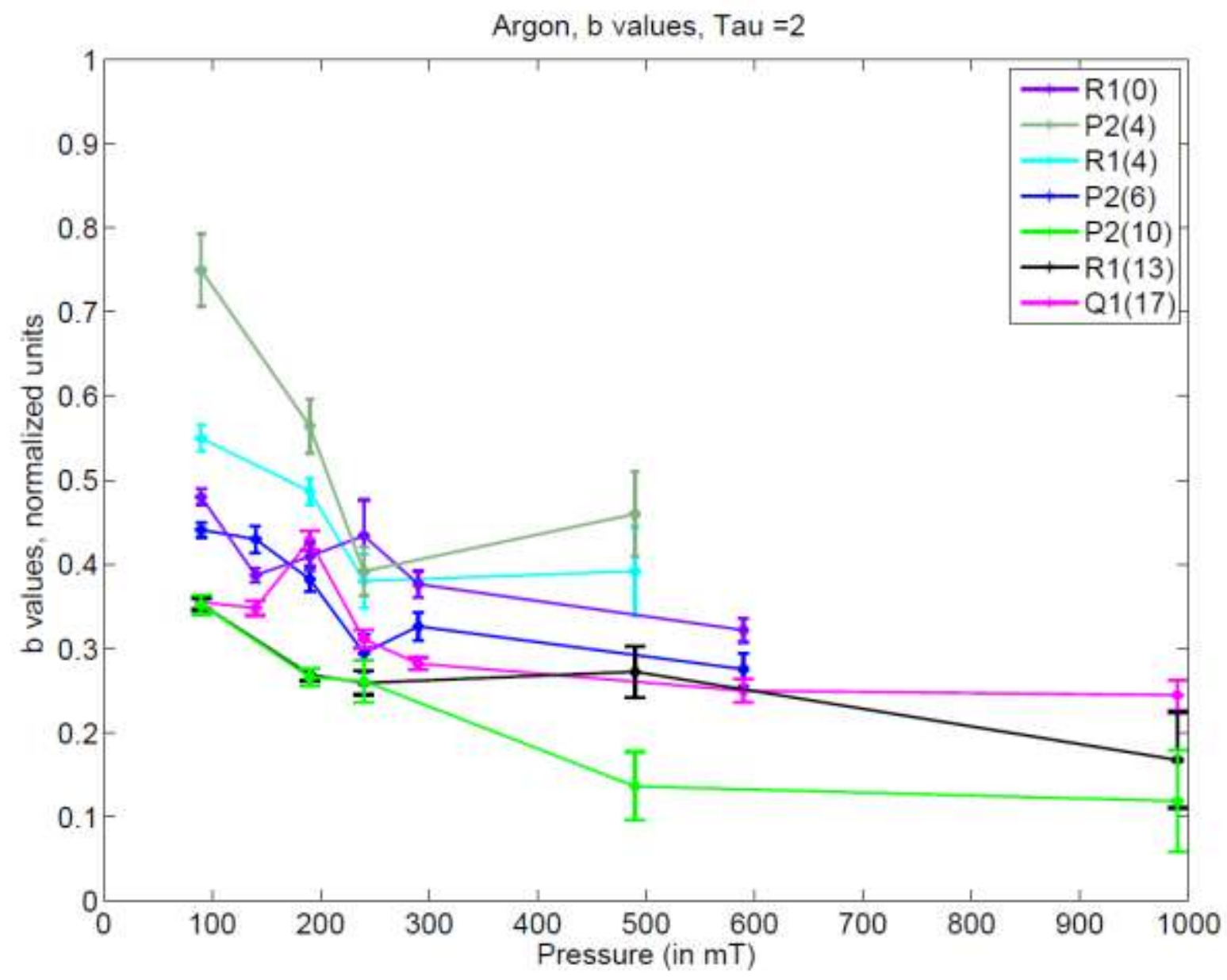

Figure S5. Argon pressure-dependent speed-dependent parameter $b$ for a selection of $\mathrm{CN}$ rotational states; $\mathrm{CH}_{3} \mathrm{COCN}$ partial pressure is $10 \mathrm{mT}$ orr for all samples, and contributes disproportionately to the speed-dependence at low total pressures. 


\section{References}

1. HIBRIDON, a package of programs for the time-independent quantum treatment of inelastic collisions and photodissociation written by M. H. Alexander, D. E. Manolopoulos, H.-J. Werner, B. Follmeg, P. J. Dagdigian, Q. Ma, and others. More information and/or a copy of the code can be obtained from the website http://www2.chem.umd.edu/groups/alexander/hibridon.

2. McGurk, S. J.; McKendrick, K. G.; Costen, M. L.; Bennett, D. I. G.; Kłos, J.; Alexander, M. H.; Dagdigian, P. J., Depolarization of rotational angular momentum in $\mathrm{CN}\left(\mathrm{A}^{2} \Pi, \mathrm{v}=4\right)+\mathrm{Ar}$ collisions. J. Chem. Phys. 2012, 136, 164306.

3. Smith, I. W. M., Kinetics and Dynamics of Elementary Gas Reactions Butterworths: London, 1980.

4. LaBudde, R. A.; Bernstein, R. B., Classical study of rotational excitation of a rigid rotor: $\mathrm{Li}^{+}+\mathrm{H}_{2}$. J. Chem. Phys. 1971, 55, 5499-5516.

5. LaBudde, R. A.; Bernstein, R. B., Classical study of rotational excitation of a rigid rotor: $\mathrm{Li}^{+}+\mathrm{H}_{2}$. II. Correspondence with quantal results. J. Chem. Phys, 1973, 59, 3687-3691.

6. Pattengill, M. D., Rotational Excitation III: Classical Trajectory Methods. In AtomMolecule Collision Theory, Bernstein, R. B., Ed. Plenum Press: New York, 1979; pp 359-375.

7. Lique, F.; Spielfiedel, A.; Feautrier, N.; Schneider, I. F.; Klos, J.; Alexander, M. H., Rotational excitation of $\mathrm{CN}(\mathrm{X})$ by He: theory and comparison with experiments. J. Chem. Phys. 2010, 132, 024303.

8. Verlet, L., Computer "Experiments" on classical fluids. I. Thermodynamical properties of Lennard-Jones molecules. Phys. Rev. 1967, 159, 98-103. 